\title{
Cell-targeted PD-1 agonists that mimic PD-L1 are potent T cell inhibitors
}

\author{
Adam P. Curnock, Giovanna Bossi, Jyothi Kumaran, Lindsay J. Bawden, Rita Figueiredo, \\ Rajeevkumar Tawar, Katherine Wiseman, Emma Henderson, Sec Julie Hoong, Veronica Conzalez, \\ Hemza Ghadbane, David E.O. Knight, Ronan O’Dwyer, David X. Overton, Christina M. Lucato, \\ Nicola M.G. Smith, Carlos R. Reis, Keith Page, Lorraine M. Whaley, Michelle L. McCully, \\ Stephen Hearty, Tara M. Mahon, and Peter Weber \\ Immunocore Ltd., Milton Park, Abingdon, United Kingdom.
}

The PD-1/PD-L1 pathway is a key immune checkpoint that regulates $\mathrm{T}$ cell activation. There is strong rationale to develop $\mathrm{PD}-1$ agonists as therapeutics against autoimmunity, but progress in this area has been limited. Here, we generated T cell receptor (TCR) targeting, PD-1 agonist bispecifics called ImmTAAI molecules that mimic the ability of PD-L1 to facilitate the colocalization of PD-1 with the TCR complex at the target cell-T cell interface. PD-1 agonist ImmTAAI molecules specifically bound to target cells and were highly effective in activating the PD-1 receptor on interacting T cells to achieve immune suppression. Potent PD-1 antibody ImmTAAI molecules closely mimicked the mechanism of action of endogenously expressed PD-L1 in their localization to the target cell-T cell interface, inhibition of proximal TCR signaling events, and suppression of $\mathrm{T}$ cell function. At picomolar concentrations, these bispecifics suppressed cytokine production and inhibited CD8 ${ }^{+} \mathrm{T}$ cell-mediated cytotoxicity in vitro. Crucially, in soluble form, the PD-1 ImmTAAI molecules were inactive and, hence, could avoid systemic immunosuppression. This study outlines a promising new route to generate more effective, potent, tissue-targeted PD-1 agonists that can inhibit T cell function locally with the potential to treat autoimmune and chronic inflammatory diseases of high unmet need.

Conflict of interest: The authors have declared that no conflict of interest exists.

Copyright: (c) 2021, Curnock et al. This is an open access article published under the terms of the Creative Commons Attribution 4.0 International License.

Submitted: June 18, 2021 Accepted: September 3, 2021 Published: October 22, 2021

Reference information: JCl Insight. 2021;6(20):e152468. https://doi.org/10.1172/jci. insight.152468.

\section{Introduction}

The PD-1 pathway is a key immune checkpoint that plays an important role in maintaining peripheral $\mathrm{T}$ cell tolerance and regulating adaptive immune responses (1-3). The PD-1 receptor can be induced on activated T cells, while its natural ligands, PD-L1 and PD-L2, are expressed by various immune and nonimmune cells (4-7). PD-1 and PD-L1 are major targets for therapeutic intervention, particularly in oncology, where several antagonistic PD-1/PD-L1 antibodies that block PD-1-mediated T cell inhibition have demonstrated efficacy in a variety of tumour types and are now approved anticancer therapies $(2,3,8)$.

In autoimmunity, there is mounting evidence that PD-1 pathway impairment plays an important role in disease pathogenesis. PD-1, PD-L1, and PD-L2 gene polymorphisms are associated with several autoimmune diseases (9-13), and perturbance of PD-1 pathway components has been reported in some indications. For example, abnormally low PD-L1 expression was noted in samples from type 1 diabetes and Crohn's disease patients (14). Additionally, PD-1 expression on effector T cells is elevated in a number of autoimmune diseases (15-17), and administration of PD-1 pathway antagonists can cause autoimmune-like symptoms in cancer patients, including aggravation of preexisting autoimmunity $(18,19)$. Together, these findings suggest that activating PD-1 on autoreactive lymphocytes may serve as a mechanism to treat autoimmune diseases. However, despite strong rationale, few PD-1 agonists have reached the clinic, and efficacy in patients is yet to be demonstrated.

One promising approach to generate efficacious PD-1 agonists is to mimic natural PD-L1-PD-1 engagement at the target cell-T cell interface. PD-L1-mediated activation of PD-1 requires accumulation of the ligand at the cell-cell interface, promoting the colocalization of PD-1 and the T cell receptor (TCR) complex at the immune synapse (20). Ligation of PD-1 enables the recruitment and activation of the SHP2 phosphatase, which in turn dephosphorylates TCR complex-signaling molecules, including ZAP-70, SLP76, and PLC $\gamma$, resulting in T cell inhibition (21-27). 
A target cell-bound PD-1 agonist - e.g., localized to pancreatic $\beta$ cells of a type 1 diabetes patient could replicate this mechanism to inhibit attacking autoreactive T cells. Such an agent would only activate the PD-1 receptor on directly interacting PD-1 ${ }^{+} \mathrm{T}$ cells and trigger localized immune suppression without impairment of the immune response in the periphery. To mimic natural PD-L1-PD-1 engagement, we engineered bispecific PD-1 agonists that can specifically bind to target cells and simultaneously activate PD-1 on interacting T cells. To do so, recombinant PD-L1 or anti-PD-1 agonist antibodies were fused to a recombinant, affinity-enhanced TCR with picomolar affinity against a target cell peptide-HLA complex. These targeted bispecifics, hereafter referred to as PD-1 agonist ImmTAAI (immune modulating monoclonal TCR against autoimmunity) molecules, effectively act as a bridge, ensuring PD-1 agonism occurs in cis with TCR engagement on the attacking autoreactive T cell.

In this study, we investigated whether target cell binding can increase the ability of PD-1 agonists to inhibit $\mathrm{T}$ cell signaling and function. We found that targeted PD-1 agonist ImmTAAI molecules are potent inhibitors of effector $\mathrm{T}$ cells, offering a promising route to successfully exploit this pathway and generate target cell-specific $\mathrm{T}$ cell inhibitors for the treatment of autoimmune diseases.

\section{Results}

Target cell-bound PD-L1 Imm TAAI molecules inhibit TCR signaling. To assess whether target cell binding is necessary for effective PD-1 agonism, PD-L1 containing bispecific ImmTAAI molecules were engineered and tested in a coculture cell assay that allowed direct comparison between target cell-bound versus unbound PD-L1 ImmTAAI. The bispecifics were designed with a targeting end composed of a soluble TCR that is highly specific for a preproinsulin (PPI) peptide, $\mathrm{PPI}_{15-24}$, bound to HLA-A*02 on pancreatic $\beta$ cells (28) and an effector end composed of either a full-length PD-L1 (flPD-L1) or a truncated PD-L1 ligand-binding domain comprising the IgV domain (IgV-PD-L1; Supplemental Figure 1A; supplemental material available online with this article; https://doi.org/10.1172/jci.insight.152468DS1). The cellular coculture assay to measure inhibition of TCR-stimulated T cell signaling was composed of an NFAT-luciferase Jurkat T cell reporter line stably expressing PD-1 (Jurkat NFL PD-1) as effector cell and an engineered HEK293T cell line expressing HLA-A2 32 -microglobulin alone or with PD-L1 as target cell (Figure 1A and Supplemental Figure 1, B and C). HEK293T-A2 target cells were also transiently transfected with membrane-bound antiCD3 antibody, bypassing the need for endogenous antigen presentation to induce $\mathrm{T}$ cell activation (hereafter referred to as HEK293T-A2/anti-CD3). Flow cytometry binding studies demonstrated that the flPD-L1 and IgV-PD-L1 ImmTAAI bispecifics bound to HEK293T-A2/anti-CD3 target cells in a concentration and peptide-dependent manner. In the presence of $\mathrm{PPI}_{15-24}$ peptide, maximum binding was achieved below 10 $\mathrm{nM}$ and $\mathrm{EC}_{50}$ values of 0.61 and $0.62 \mathrm{nM}$, for flPD-L1 and IgV-PD-L1 ImmTAAI, respectively (Figure 1B).

Coculture of effector and target cells resulted in measurable NFAT activity (Supplemental Figure 1D), while overexpression of PD-L1 on the HEK293T-A2/anti-CD3 target cells resulted in reduction of NFAT activity by more than $75 \%$, highlighting that $\mathrm{T}$ cell activation in this assay is sensitive to the PD-1/PD-L1 pathway. In the absence of PD-L1 expression, both flPD-L1 and IgV-PD-L1 ImmTAAI molecules inhibited NFAT activity in a concentration-dependent manner when added to cocultures of effectors and PPI ${ }_{15-24}$ peptide-pulsed target cells (Figure 1, C and D). However, soluble flPD-L1-Fc was completely inactive in this assay, as was $\mathrm{PPI}_{15-24}$ TCR alone (Figure $1 \mathrm{D}$ and Table 1). The IgV-PD-L1 ImmTAAI was more effective than the flPD-L1 at inhibiting NFAT activity in pulsed cells (inhibition down to 35\% versus 58\% NFAT activity at $100 \mathrm{nM} ; P<0.001$ ), with efficacy similar to that observed with PD-L1-expressing HEK293T-A2/ anti-CD3 target cells (Supplemental Figure 1D), while neither ImmTAAI molecule inhibited NFAT activity without $\mathrm{PPI}_{15-24}$ peptide pulsing of the target cells. Thus, flPD-L1 and IgV-PD-L1 ImmTAAI molecules can inhibit $\mathrm{T}$ cells in a target-dependent fashion but only when bound to the target cell, mimicking natural PD-L1-PD-1 engagement.

PD-1 antibody Imm TAAI molecules are more potent inhibitors of T cell activation than PD-L1 ImmTAAI molecules. Antibody-based agents possess a number of potential advantages over recombinant PD-L1, including enhanced stability and scope for improved potency, in the generation of clinical therapeutics. As such, we identified several single variable domain on a heavy chain (VHH) PD-1 antibodies with low nanomolar affinities and fused these to the $\mathrm{PPI}_{15-24}$ TCR to produce a panel of PD-1 agonist ImmTAAI molecules. The cellular potency of these antibody-based bispecifics was then compared with their PD-L1-containing counterparts in the HEK293T-A2-Jurkat NFL PD-1 reporter assay. Like the PD-L1 ImmTAAI molecules, each of the VHH-based PD-1 agonist ImmTAAI molecules dose 
A
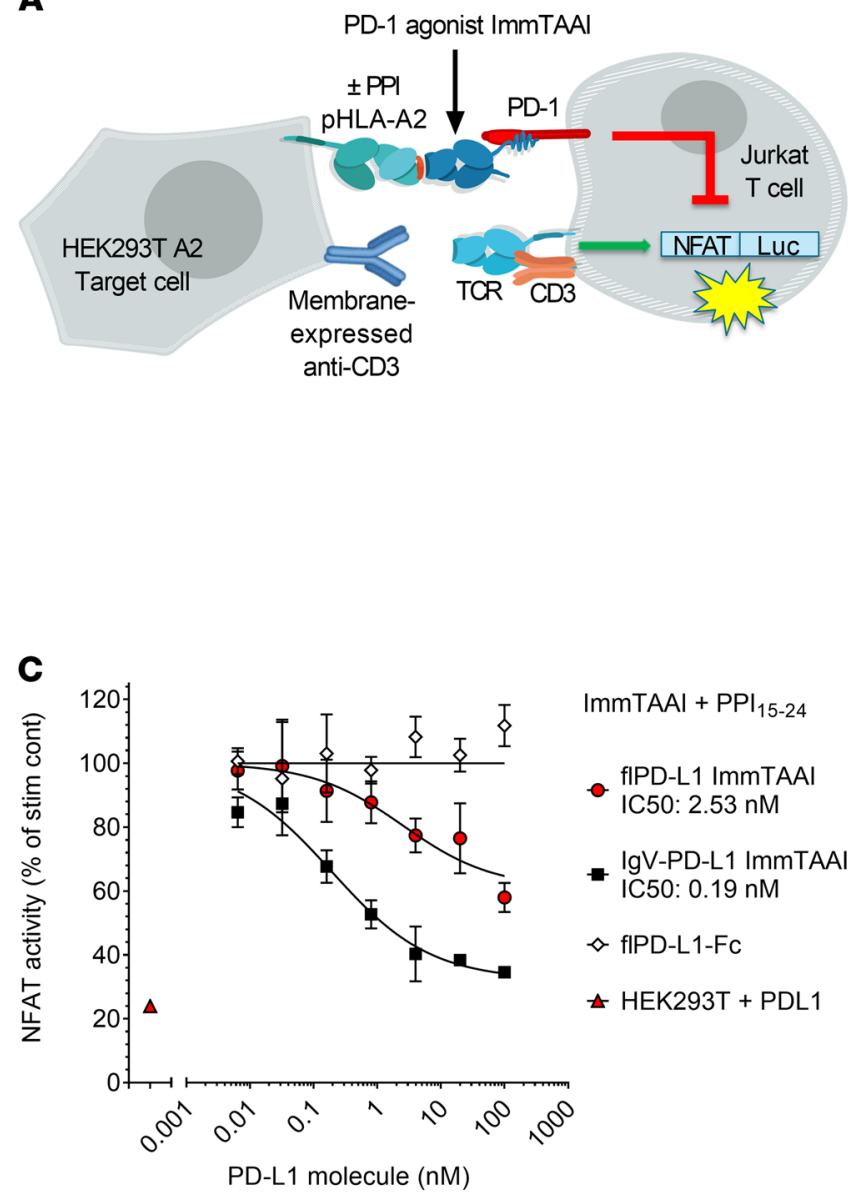
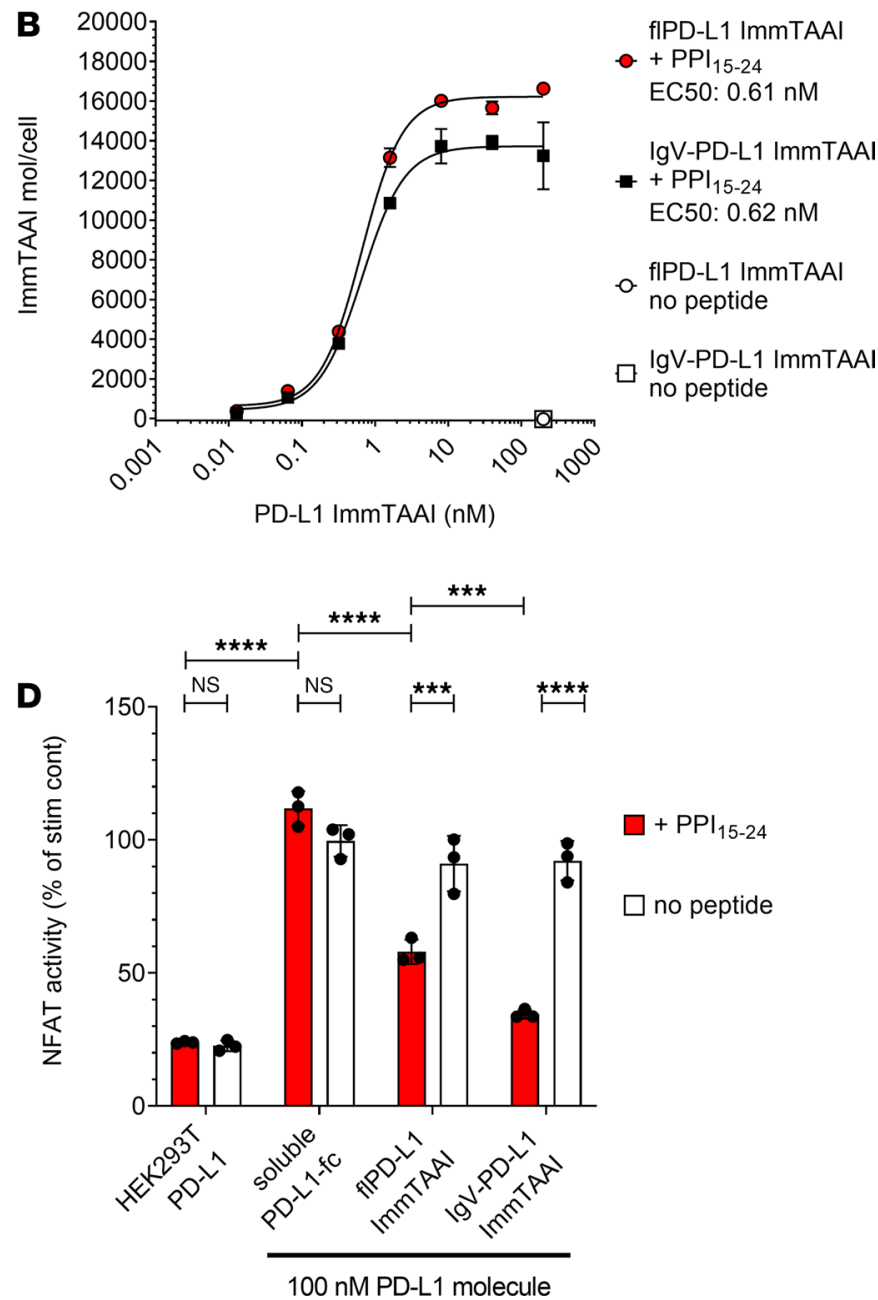

Figure 1. Target cell-bound PD-L1 ImmTAAI molecules inhibit TCR complex signaling. (A) Schematic of the HEK293T-A2-Jurkat NFL PD-1 reporter assay. (B) PD-L1 ImmTAAI titrations were incubated with PPI $_{15-24}$ peptide-pulsed or nonpulsed HEK293T-A2 target cells. ImmTAAI binding was quantified by flow cytometry, and dose-response curves were plotted ( $n=3$ and representative of 3 independent experiments). (C) Dose responses of the PD-L1 ImmTAAIs and PD-L1 Fc were tested in the HEK293T-A2-Jurkat NFL PD-1 reporter assay. Normalized NFAT activity was plotted against ImmTAAI concentration to calculate $\mathrm{IC}_{50}$ values ( $n=3$ and representative of 3 independent experiments). (D) Relative NFAT activity at $100 \mathrm{nM}$ ImmTAAI was plotted for pulsed ( $+\mathrm{PPI}_{15-24}$ ) versus nonpulsed (no peptide) target cells ( $n=3$ and representative of 3 independent experiments). All data are plotted as mean \pm SD and were compared by 2-way ANOVA with repeated measures and Tukey's or Sidak's multiple-comparison test. ${ }^{* * *} P \leq 0.001,{ }^{* * * *} P \leq 0.0001$. IgV, immunoglobulin-like variable domain; fl, full-length.

dependently inhibited NFAT activity (Figure 2A), measured using the NFAT-luciferase reporter assay described in Figure 1. To explore an alternative antibody format, a single-chain Fv (scFv) fragment (CA949) PD-1 agonist antibody (29) was fused to the PPI ${ }_{15-24}$ TCR. This ImmTAAI caused NFAT inhibition comparable with that observed with VHH-based ImmTAAI molecules. This inhibitory effect was dependent on target cell binding, since no inhibition occurred with soluble VHH or scFv (CA949) antibodies alone or in the absence of peptide (Figure 2B and Table 1).

From the selection of VHH antibodies, $\mathrm{H} 5$ was chosen for further studies, as it displayed better potency in some assays. In a direct comparison with the IgV-PD-L1 ImmTAAI, the antibody-based bispecifics H5 and CA949 ImmTAAI exhibited superior potency and efficacy in inhibiting TCR-stimulated NFAT activity in cocultures with peptide-pulsed target cells (Figure 2, C and D, and Table 1). This indicates that bispecific molecules composed of an affinity-enhanced TCR targeting moiety and a potent PD-1 agonist antibody can mimic cellular PD-L1 in promoting PD-1-mediated suppression of $\mathrm{T}$ cell activation, providing increased potency compared with the natural ligand.

ImmTAAI molecules are designed to inhibit $\mathrm{T}$ cell activation through agonism of the PD-1 pathway — but only when the $\mathrm{T}$ cell is in close proximity to the targeted cell type. To ensure that the observed 
Table 1. HEK293T-A2-Jurkat NFL PD-1 reporter assay data summary.

\begin{tabular}{lcc}
\hline Test molecule & Max inhib \% (SD, $\boldsymbol{n})$ & IC50 $\mathbf{n M}(\mathbf{S D}, \boldsymbol{n})$ \\
PD-L1 O/E HEK293T cells & $80.1(7.1,10)$ & NA \\
IgV-PD-L1 ImmTAAI & $52.0(7.3,7)$ & $0.374(0.189,7)$ \\
fIPD-L1 ImmTAAI $^{\text {fIPD-L1-Fc }}$ & $35.7(8.5,3)$ & $1.38(1.63,3)$ \\
PPI $_{15-24}$ TCR & $<10(\mathrm{NA}, 4)$ & $>100(\mathrm{NA}, 4)$ \\
VHH A6 ImmTAAI $_{\text {VHH G1 ImmTAAI }}$ & $<10(\mathrm{NA}, 4)$ & $>100(\mathrm{NA}, 4)$ \\
VHH E6 ImmTAAI $_{\text {VHH H2 ImmTAAI }}$ & $63.7(4.2,3)$ & $0.187(0.092,3)$ \\
VHH H5 ImmTAAI & $66(8.2,3)$ & $0.384(0.331,3)$ \\
scFv CA949 ImmTAAI & $71.3(6.1,3)$ & $0.127(0.034,3)$ \\
VHH H5 Ab & $64.3(11.2,3)$ & $0.089(0.067,3)$ \\
scFv CA949 Ab & $74.7(4.5,6)$ & $0.094(0.036,6)$ \\
\hline
\end{tabular}

Maximal percent inhibition and $I_{50}$ values for Jurkat NFL PD-1 NFAT activity at 16 hours. NA, not applicable; $0 / E$, overexpressing; scFv, single-chain fragment variable; $A b$, antibody.

PD-1 agonist activity in a cell-bound state was not unique to the PPI ${ }_{15-24}$ TCR targeting moiety, IgVPD-L1 and CA949 were each fused to an alternate affinity-enhanced TCR specific for gp $100_{280-288}$ (HLA-A*02:01, YLEPGPVTA), a melanoma epitope (30). Altering TCR specificity had no effect on the PD-1 agonist activity of the ImmTAAI, since each gp100-targeted molecule dose-dependently inhibited TCR-stimulated NFAT activation to a similar level as their PPI-targeted equivalent (Figure 2E), but only when target cells were pulsed with their specific peptide (Figure $2 \mathrm{~F}$ ). These data indicate that, although target cell binding via the TCR targeting domain is essential for ImmTAAI-mediated PD-1 agonism, the incorporated TCR can be varied to achieve specificity for different cell types or tissues, assuming the target epitope is presented in sufficient quantities.

To further characterize our ImmTAAI molecules, we designed an alternative NFAT reporter assay incorporating cells with natural peptide HLA ( $\mathrm{pHLA}$ ) target presentation and TCR recognition of cognate antigen. To test whether endogenous levels of $\mathrm{PPI}_{15-24}$-HLA-A2 presentation are sufficient to trigger ImmTAAI-mediated PD-1 agonism, the $\beta$ cell line ECN90, which naturally processes and presents the PPI $_{15-24}$ peptide-HLA-A2 complex, was used as target cells (Figure 3A). Full-length and truncated PD-L1 ImmTAAI molecules specifically bound ECN90 target cells with a similar $\mathrm{EC}_{50}$ as that observed for the peptide-pulsed HEK293T-A2 target cells in Figure 1, albeit with a lower total occupancy reflecting the lower natural pHLA levels (Figure 3B).

To provide TCR activation via recognition of cognate antigen, the Jurkat NFL/PD-1 effector cells were transduced with a Mel5 TCR that recognizes the Melan- 126-135 $_{1}$ (HLA-A*02:01, ELAGIGLTV) epitope (Supplemental Figure 2A). ECN90 target cells were pulsed with Melan- $\mathrm{A}_{126-135}$ peptide, enabling physiological T cell activation via natural TCR engagement. NFAT activity downstream of TCR engagement in the TCR transgenic Jurkat effector cell line was efficiently suppressed when cocultured with ECN90 target cells that were induced to overexpress PD-L1 (Supplemental Figure 2, B and C), confirming functionality of this in vitro assay.

In coculture with Melan-A peptide-pulsed ECN90 target cells, the flPD-L1 and IgV-PD-L1 ImmTAAI, as well as the antibody-based PD-1 ImmTAAI molecules, inhibited TCR complex signaling to a similar extent as PD-L1-expressing ECN90 cells (Figure 3, C and D, and Table 2). Consistent with results using peptide-pulsed target cells, the flPD-L1 ImmTAAI showed that the weakest inhibitory activity and soluble forms of each molecule (lacking the TCR targeting domain) failed to inhibit NFAT activity (Figure 3, C and D). Again, antibody-based PD-1 agonist ImmTAAI molecules were more potent than PD-L1-containing bispecifics. Together, these data show that, in a system using physiological $\mathrm{T}$ cell activation, target cell-bound antibody-based PD-1 agonists are as effective as natural PD-L1 in inhibiting TCR complex signaling in T cells.

$P D-1$ antibody ImmTAAI molecules are noncompetitive with PD-L1 for PD-1 binding and are additive with $P D-L 1$ in suppressing TCR complex signaling. Antibodies to PD-1 that do not compete with PD-L1 binding are advantageous for the design of PD-1 agonist T cell inhibitors as they potentially avoid interfering with physiological PD-L1 activity and may even enhance T cell suppression in the presence of PD-L1-expressing 
A

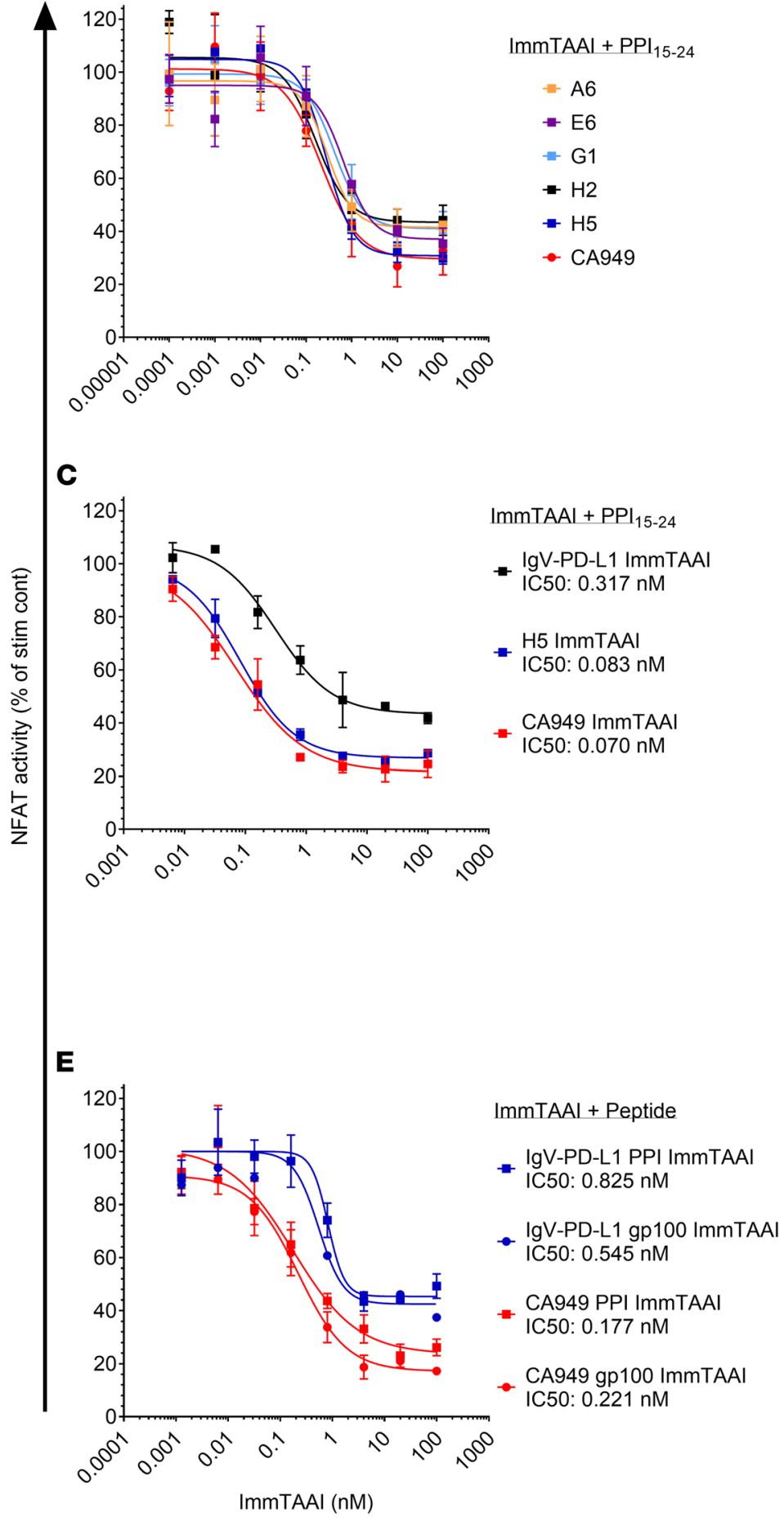

B

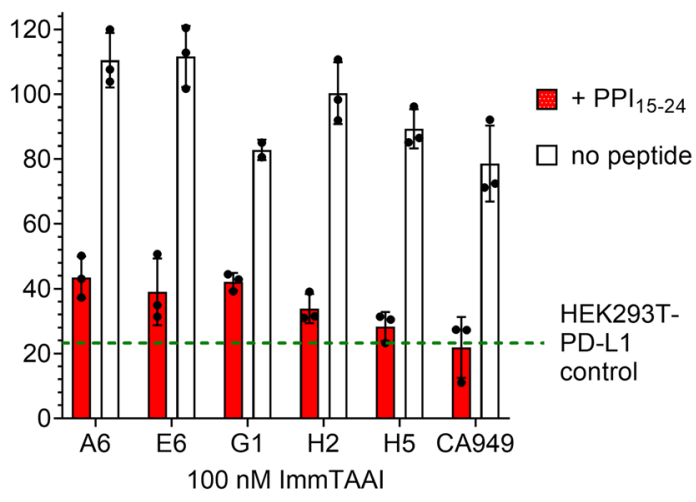

D

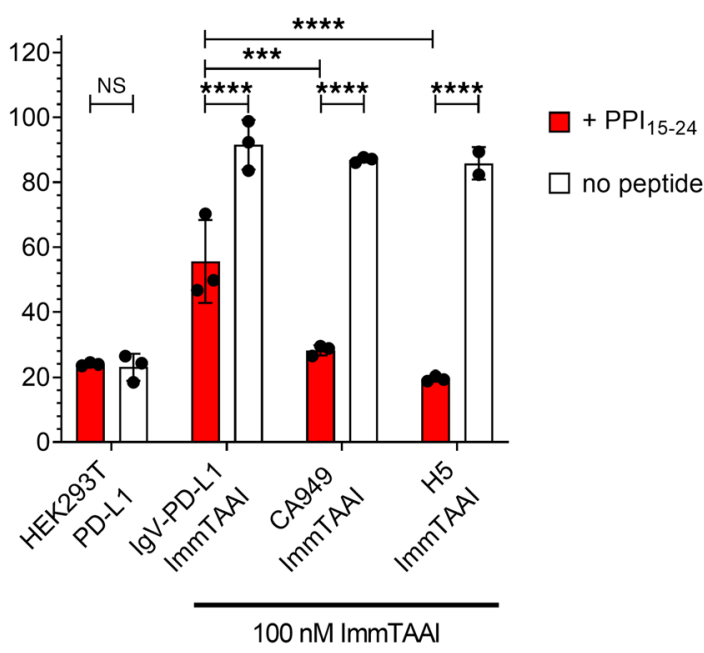

$\mathbf{F}$

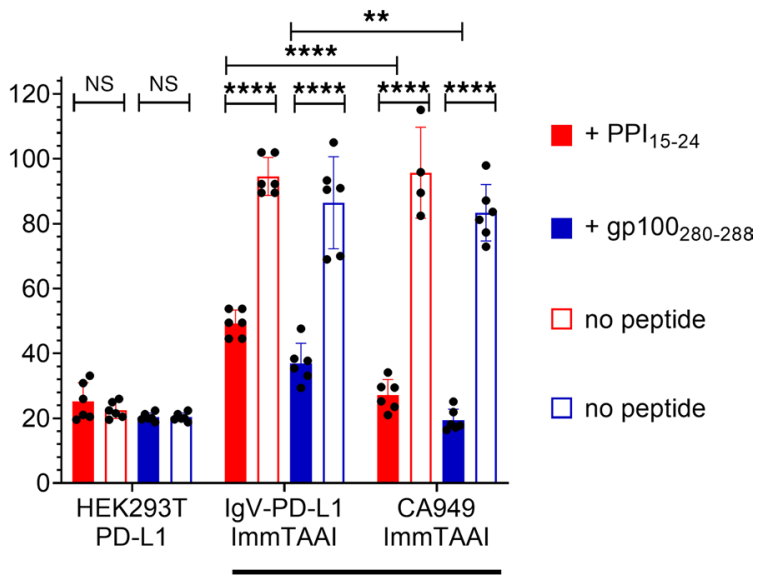

Figure 2. Target cell-bound PD-1 antibody ImmTAAI molecules exhibit superior inhibition of TCR complex signaling over PD-L1 ImmTAAI molecules. (A and B) PD-1 antibody ImmTAAI molecules constructed using a panel of VHH PD-1 antibodies and an SCFv PD-1 antibody (CA949) were tested in the HEK293T-A2/anti-CD3: Jurkat NFL PD-1 reporter assay as described in Figure 1 ( $n=3$ and representative of 3 independent experiments). (C and D) Representative VHH-based (H5) and scFv-based (CA949) ImmTAAI molecules were tested alongside the IgV-PD-L1 ImmTAAI in the HEK293T-A2/anti-CD3: Jurkat NFL PD-1 reporter assay, and data are plotted as described above ( $n=3$ and representative of 3 independent experiments). (E and F) PD-1 agonist ImmTAAI molecules were generated by fusing CA949 scFV antibody and IgV-PD-L1 to different TCRs against either gp100 ${ }_{280-288} \mathrm{PHLA}^{-A 2}$ or PPI ${ }_{15-24}$ pHLA-A2. The ImmTAAI molecules were tested in the HEK293T-A2/anti-CD3: Jurkat NFL PD-1 reporter assay, using HEK293T-A2 target cells pulsed with the appropriate targeting peptide ( $n=6$ and representative of 2 independent experiments). All data are plotted as mean \pm SD and were compared by 2 -way ANOVA with repeated measures and Tukey's or Sidak's multiple-comparison test. ${ }^{* *} P \leq 0.01,{ }^{* *} P \leq 0.001,{ }^{* * *} P \leq 0.0001$. 
A

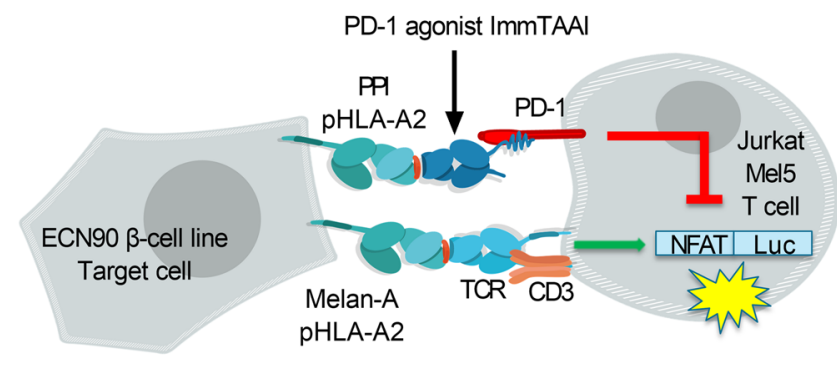

B
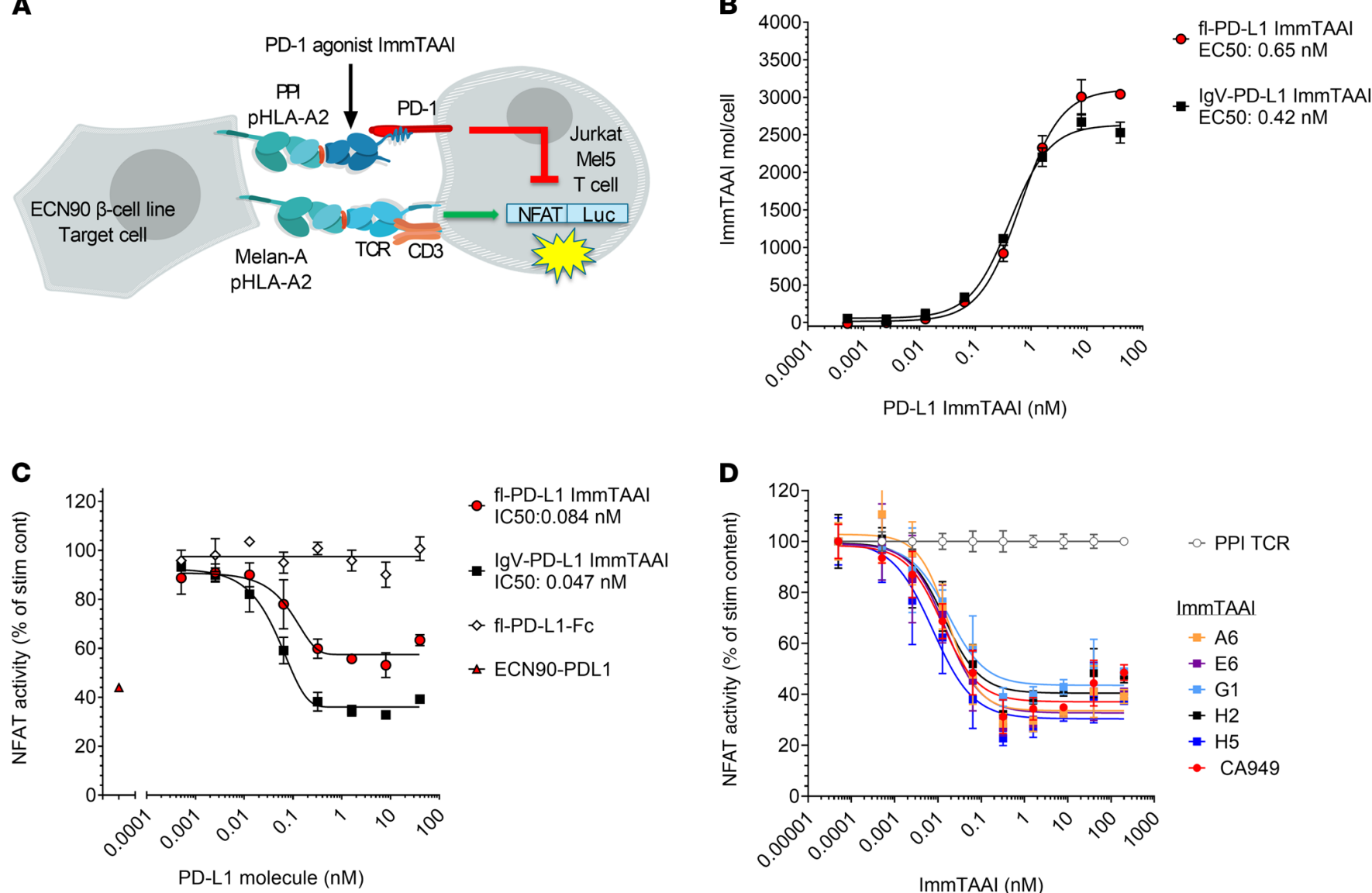

D

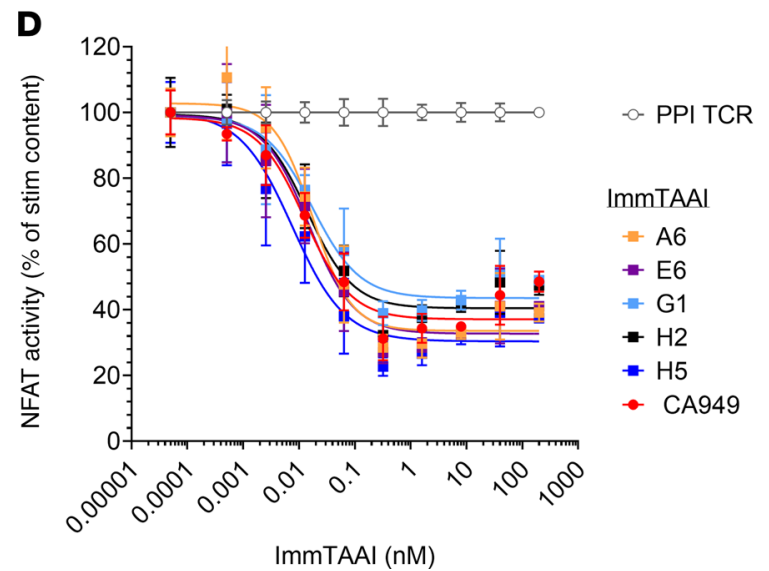

Figure 3. $\beta$ Cell-targeted PD-1 agonist ImmTAAI molecules inhibit TCR complex signaling. (A) Schematic of the ECN90 $\beta$ cell line: Jurkat NFL Mel5 PD-1 reporter assay. ECN90 cells were pulsed with Melan-A activating peptide, and titrations of PD-L1 or PD-1 Ab ImmTAAI molecules, PD-L1 Fc, or PPI TCR alone added. (B-D) Either ECN90 cells were incubated for 3 hours and analyzed for PD-L1 ImmTAAI binding ( $n=3$ and representative of 3 independent experiments) (B), or Jurkat NFL Mel5 PD-1 cells were immediately added to test activity in the PD-1 reporter assay ( $n=3$ and representative of 3 independent experiments) (C and D). All data are plotted as mean \pm SD. Abbreviations: IgV, Immunoglobulin-like variable domain; fl, full length

target cells. In a competition binding study using surface plasmon resonance (SPR; Supplemental Figure 3), neither the CA949 nor the H5 PD-1 antibody ImmTAAI competed with PD-L1 for PD-1 binding (Figure 4, A and B), presenting the possibility that addition of PD-1 antibody ImmTAAI molecules could enhance PD-L1-mediated T cell inhibition.

To investigate if these bispecifics could enhance PD-L1-driven T cell inhibition, increasing concentrations of either the H5 or CA949-ImmTAAI molecules were added to Jurkat NFL Mel5 PD-1 effector cells cultured with PD-L1-transfected ECN90 target cells. In the absence of ImmTAAI molecules, PD-L1 expression in the ECN90 cells inhibited Mel5 TCR-stimulated NFAT activity by $60 \%-75 \%$ compared with the PD-L1- ECN90 parental line (Figure 4C; top curves). Addition of either the H5 or CA949 PD-1 antibody ImmTAAI molecules to this system dose-dependently enhanced PD-L1-mediated inhibition of NFAT activity, with $\mathrm{IC}_{50}$ values of approximately $25 \mathrm{pM}$. The combined activities of cellular PD-L1 and PD-1 antibody ImmTAAI inhibited NFAT activation by greater than $90 \%$ (Figure 4C; bottom curves). Notably, the $\mathrm{IC}_{50}$ values of the PD-1 antibody ImmTAAI molecules required here were the same as those observed in the absence of PD-L1 expression (Figure 4C; top curves), indicating that this effect was additive rather than synergistic. In contrast, a PD-L1-containing ImmTAAI had no significant effect on PD-L1-mediated suppression of NFAT activity (Figure 4D). The added inhibition conferred by the PD-1 antibody ImmTAAI is also unlikely due to engagement of additional free PD-1, since the level of PD-L1 expression on the ECN90 $\beta$ cell line is well in excess of PD-1 expression on the Jurkat T cells (Supplemental Figure 2A and Supplemental Figure 1C).

Conversely, to rule out that the ImmTAAI molecules function as blocking antibodies to PD-L1 in the unbound state, the $\mathrm{H} 5 \mathrm{VHH}$ and CA949 $\mathrm{scFv}$ were fused to an irrelevant gp100 TCR that does 
Table 2. ECN90-Jurkat NFL Mel5 PD-1 reporter assay data summary.

\begin{tabular}{lcc}
\hline Test molecule & Max inhib \% (SD, $\boldsymbol{n})$ & IC50 $\mathbf{n M}(\mathbf{S D}, \boldsymbol{n})$ \\
PD-L1 O/E HEK293T cells & $70.6(15.2,7)$ & NA \\
IgV-PD-L1 ImmTAAI & $62.0(8.7,3)$ & $0.047(0.01,3)$ \\
fIPD-L1 ImmTAAI $^{\text {fIPD-L1-Fc }}$ & $40.3(5.8,3)$ & $0.165(0.147,3)$ \\
PPI $_{15-24}$ TCR & $<10(\mathrm{NA}, 3)$ & $>100(\mathrm{NA}, 3)$ \\
VHH A6 ImmTAAI $_{\text {VHH G1 ImmTAAI }}$ & $<10(\mathrm{NA}, 3)$ & $>100(\mathrm{NA}, 3)$ \\
VHH E6 ImmTAAI $_{\text {VHH H2 ImmTAAI }}$ & $71.7(2.2,3)$ & $0.023(0.015,3)$ \\
VHH H5 ImmTAAI & $61.7(2.6,3)$ & $0.026(0.009,3)$ \\
scFv CA949 ImmTAAI & $72.7(2.9,3)$ & $0.023(0.015,3)$ \\
VHH H5 Ab & $71.4(3.9,3)$ & $0.017(0.012,3)$ \\
scFv CA949 Ab & $77.0(3.7,3)$ & $0.017(0.011,3)$ \\
\hline
\end{tabular}

Maximal percent inhibition and IC ${ }_{50}$ values for Jurkat NFL Mel5 PD-1 NFAT activity at 16 hours. NA, not applicable; $0 / E$, overexpressing; scFv, single-chain fragment variable; $A b$, antibody.

not recognize peptide-HLA on the surface of ECN90 cells. Both gp100-targeted H5 and CA949 PD-1 antibody ImmTAAI molecules were unable to either interfere with or enhance PD-L1-mediated T cell inhibition in an unbound, soluble state, while pembrolizumab, a well characterized PD-1 antagonist antibody, potently antagonized PD-L1-mediated suppression of TCR-stimulated NFAT activity (Figure $4 \mathrm{E})$. Together, these results highlight the potential for cell-bound PD-1 antibody ImmTAAI molecules to enhance PD-1-mediated suppression of $\mathrm{T}$ cell responses in the presence of endogenous PD-L1.

PD-1 antibody Imm TAAI molecules colocalize with PD-1 at the target cell-effector cell interface and inhibit proximal TCR signaling events. Cellular PD-L1 accumulates at the target cell-T cell interface upon engagement of PD-1 and promotes PD-1/TCR coclustering on the T cell $(20,23)$. To assess whether the PD-1 agonist ImmTAAI molecules also localize to the T cell-target cell interface, we conducted immunofluorescence studies with labeled ImmTAAI in ECN90/Jurkat Mel5 cocultures. The H5 PD-1 antibody ImmTAAI was fluorescently labeled with Alexa Fluor 488 (AF488), and localization was analyzed by confocal microscopy in cocultures with Melan-A peptide-pulsed ECN90 $\beta$ cell targets and either PD-1-transduced or nontransduced Jurkat Me15 effector cells (Figure 5A). The AF488-ImmTAAI bound to the ECN90 target cells and accumulated at the cell-cell interface in cultures with PD-1expressing Jurkat Me15 cells in 87\% of captured conjugation events (Figure 5, B and C). However, in the absence of PD-1 expression, the ImmTAAI remained dispersed over the surface of the $\beta$ cell line, and accumulation was not observed for any Jurkat Mel5-target cell conjugates (Figure 5C). This suggests that, as with cellular PD-L1, a targeted synthetic PD-1 agonist accumulates at the T cell-target cell interface upon engagement with PD-1.

We next assessed how this relocalization and accumulation of PD-1 to cell-cell interface upon engagement with target cell-bound ImmTAAI affected proximal TCR signaling. Coculturing the Mel5 TCR transgenic Jurkat T cells with PD-L1-expressing ECN90 target cells resulted in a marked decrease in the phosphorylation of SLP-76, PLC $\gamma$, and ZAP-70 in the responding effector T cells, as previously reported (Figure $5 \mathrm{D}$ and Supplemental Figure 4, A-C) $(23,25)$. A similar pattern and extent of inhibition was observed when target cells were preloaded with either the H5 or the CA949 ImmTAAI molecules (Figure 5E and Supplemental Figure 4, D-I), indicating that the PD-1 agonist ImmTAAI molecules are as effective as PD-L1 in inhibiting proximal TCR signaling events. These findings further support the hypothesis that target cellbound PD-1 antibody ImmTAAI molecules mimic the natural ligand, both in terms of their accumulation at the target cell-T cell interface and their ability to inhibit signaling downstream of TCR engagement.

PD-1 antibody ImmTAAI molecules inhibit activation of primary human $C D 4^{+} T$ cells by antigen-presenting cells. The priming of autoreactive $\mathrm{CD} 4^{+} \mathrm{T}$ cells by antigen-presenting cells (APCs) is a key event in many autoimmune diseases. To assess if APC-targeted PD-1 ImmTAAI molecules could provide benefits in such a setting, we generated an assay to determine the impact of these bispecifics on the activation of primary 
A

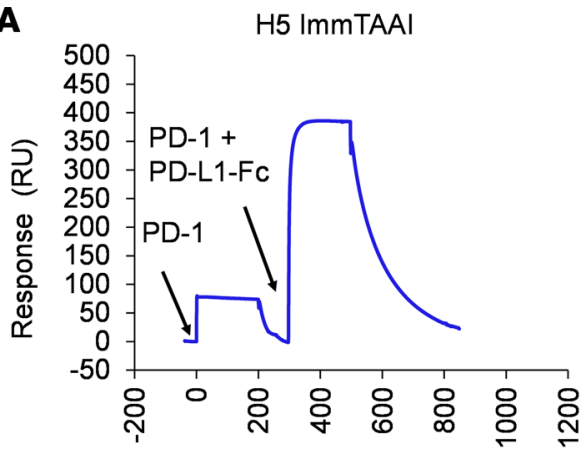

Time (s)

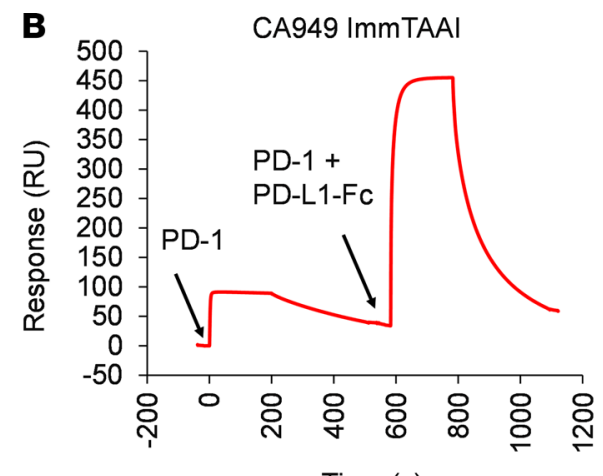

Time (s)

\section{C}
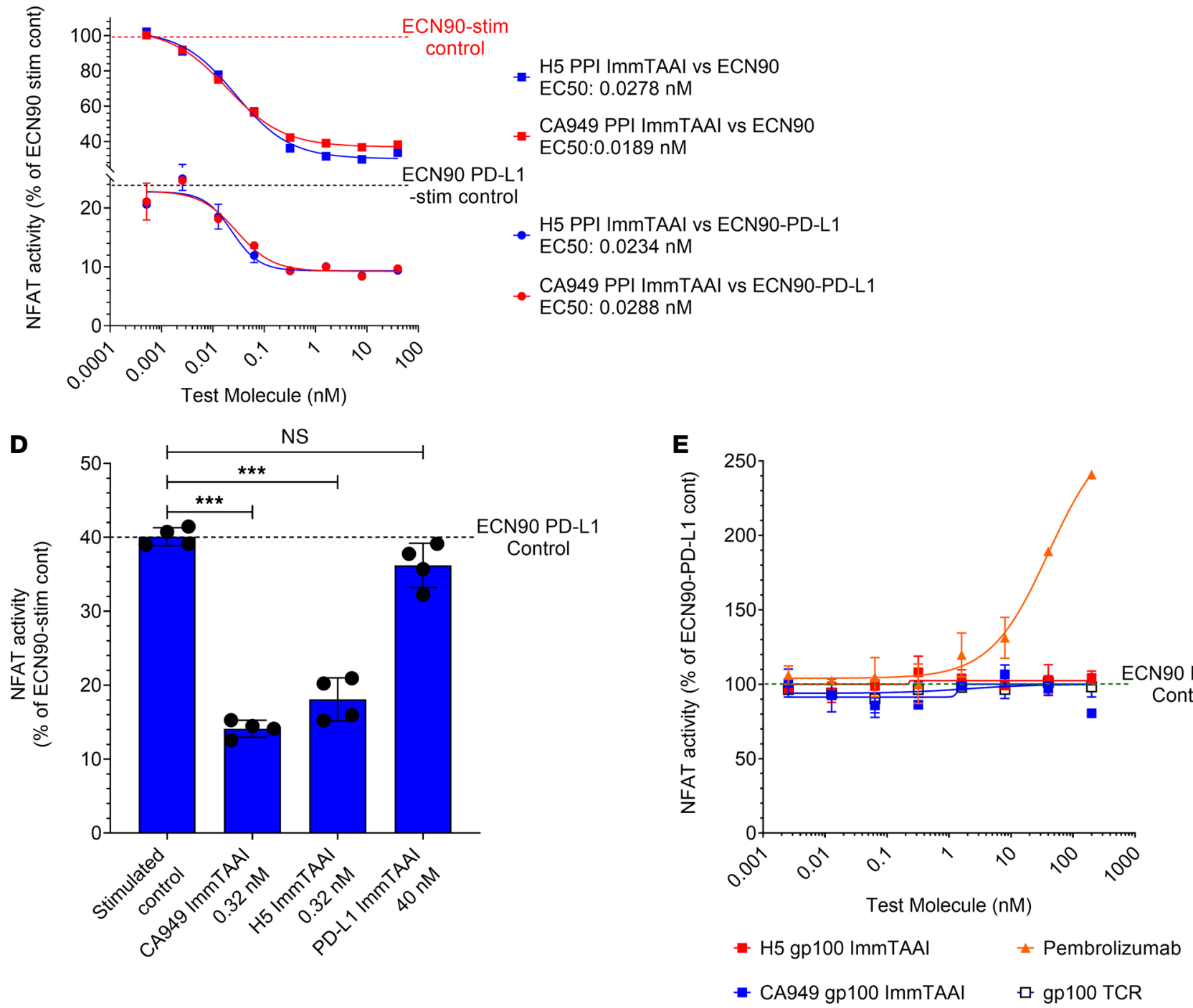

Figure 4. PD-1 antibody ImmTAAI molecules are noncompetitive with PD-L1 for PD-1 binding and are additive with PD-L1 in inhibiting TCR complex signaling. (A and B) SPR competition binding studies were conducted as described in Methods and Supplemental Figure 3. Response units (RU) were plotted over time to characterize the binding of H5- and CA949-ImmTAAI molecules to PD-1 in the presence of PD-L1-Fc (representative sensorgrams from 3 independent experiments). (C) Parental ECN90 (upper curves) or PD-L1 transduced ECN90 cells (lower curves) were pulsed with Melan-A activating peptide and titrations of PD-1 antibody ImmTAAI molecules added. Jurkat NFL Mel5 PD-1 cells were immediately added to run the PD-1 reporter assay ( $n$ $=2$ and representative of 4 independent experiments). (D) Cell assay using PD-L1 transduced ECN90 cells was done as described above using titrations of PD-1 antibody ImmTAAI or PD-L1 ImmTAAI molecules. Relative NFAT activity is plotted against the concentrations where maximal inhibition was observed ( $n=4$ and representative of 4 independent experiments). (E) Titrations of soluble PD-1 antibody ImmTAAI molecules (with an irrelevant TCR) and a PD-1 blocking antibody (pembrolizumab) were tested in the ECN90-PD-L1: Jurkat NFL Mel5 PD-1 reporter assay ( $n=2$ and representative of 2 independent experiments). Data are plotted as mean \pm SD and were compared by 1-way ANOVA and Dunnett's multiple-comparison test. ${ }^{* *} P \leq 0.001$. 
A

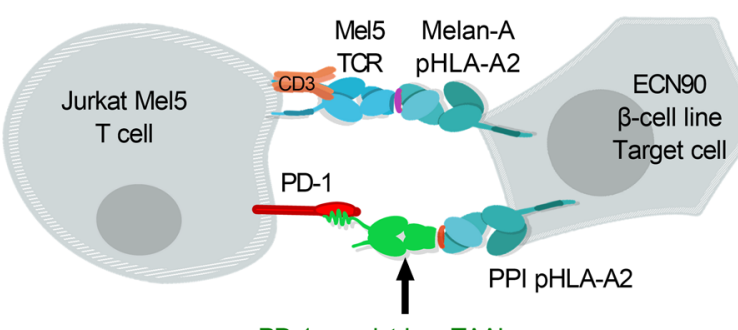

PD-1 agonist ImmTAAI
B

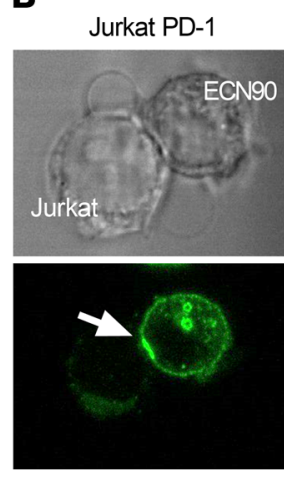

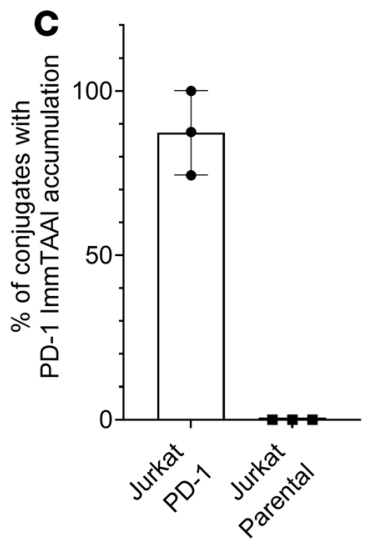
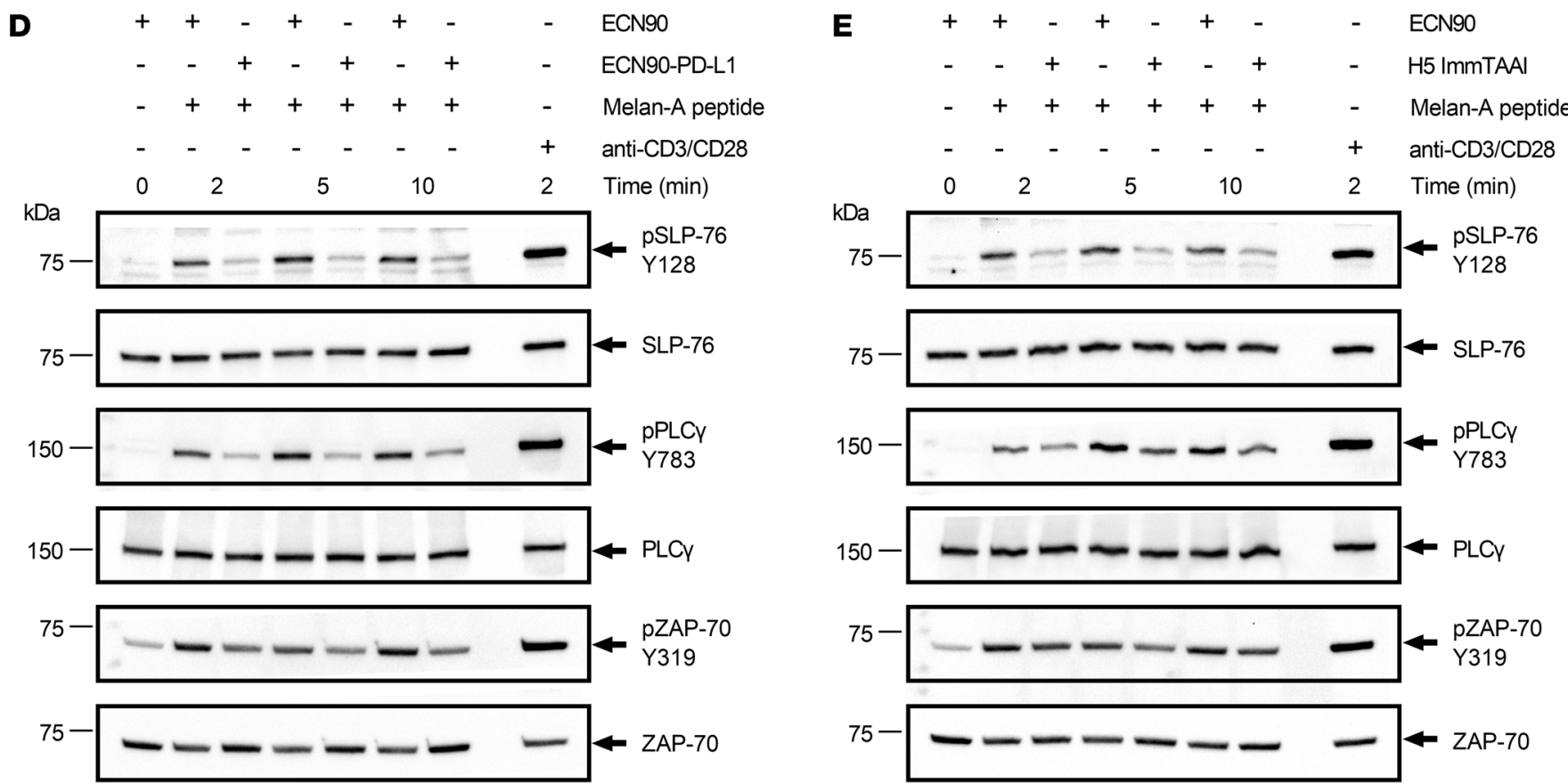

Figure 5. H5 ImmTAAI localizes at the target cell-T cell interface and inhibits proximal TCR signaling. (A) Schematic of the cell assay used to visualize $\mathrm{H} 5$ ImmTAAl localization. (B) Phase contrast and confocal fluorescence images of H5 ImmTAAl localization on ECN90 $\beta$ cell target cells cocultured with either PD-1+ or PD-1- Jurkat cells. Upper panel: phase contrast. Lower panel: fluorescence images, a white arrow indicates ImmTAAI accumulation (representative images from 3 independent studies). Total original magnification, $\times 100$. (C) The numbers of ECN90 $\beta$ cell: Jurkat conjugates with ImmTAAI accumulation (white arrow) were counted and plotted as a percentage of the total number of cell conjugates ( $n=100$ conjugates for conditions using Jurkat Mel5 PD-1 cells and $n=96$ for Jurkat Mel5 cells, plotted as mean data from 3 independent studies). (D) Jurkat Mel5 PD-1 cells were stimulated with Melan-A-pulsed ECN90 or ECN90 PD-L1 cells for the indicated times. Whole cell lysates for each condition and time point were resolved by SDS-PAGE, transferred to PVDF membranes, and probed by Western blotting for phospho-SLP-76 (pSLP-76), total SLP-76 (SLP-76), phospho-PLC $\gamma$ (pPLC $\gamma$ ), total PLC $\gamma$ (PLC $\gamma$ ), phospho-ZAP-70 (pZAP-70), and total ZAP-70 (representative blots from 3 independent experiments). (E) Jurkat Mel5 PD-1 cells were stimulated with Melan-A-pulsed ECN9O cells, in the presence or absence of H5 ImmTAAl, and Western blots were performed as above (representative blots from 3 independent experiments).

$\mathrm{CD}^{+} \mathrm{T}$ cells. Raji cells, which naturally express the CD28 ligands CD80 and CD86 (Supplemental Figure $5 \mathrm{~A}$ ), were used as APCs and transduced with an HLA-A2 $\beta 2$-microgobulin lentivirus (Raji-A2) to enable $\mathrm{PPI}_{15-24}$ peptide presentation and ImmTAAI binding (Figure 6A). For effector cells, primary human T cells, isolated from PBMC, were first stimulated with superantigen-loaded (SEB-loaded) Raji-A2 APCs to induce PD-1 expression (Supplemental Figure 5, B and C). Preactivated CD $4^{+} \mathrm{PD}-1^{+} \mathrm{T}$ cells were subsequently restimulated with unpulsed or PPI 15-24 $_{24}$ peptide-pulsed SEB-loaded Raji-A2 cells to assess PD-1 agonist ImmTAAI activity in either APC-bound or soluble form. In the presence of $\mathrm{PPI}_{15-24}$ peptide-pulsed APCs, all PD-1 agonist ImmTAAI molecules reduced IL- 2 production by $40 \%-50 \%$ from activated T cells at 
picomolar levels but were inactive in the presence of nonpulsed APCs (Figure 6, B and C, and Table 3). As in previous assays, the antibody-based ImmTAAI molecules were more potent and efficacious than PD-L1 ImmTAAI molecules, and nontargeted soluble antibodies or antibodies fused to an irrelevant TCR failed to inhibit IL-2 production (Figure 6, B and C). Together, these data build on our findings from reporter-based cell systems and demonstrate that cell-targeted PD-1 agonist ImmTAAI molecules are potent inhibitors of primary $\mathrm{CD}^{+} \mathrm{T}$ cell activation.

PD-1 antibody Imm TAAI molecules inhibit autoreactive human $C D 8^{+} T$ cell activation and protect target cells from $T$ cell killing. To explore if PD-1 agonist ImmTAAI molecules can inhibit autoreactive CD8 ${ }^{+} \mathrm{T}$ cells, a T cell cytotoxicity assay was developed using the pancreatic $\beta$ cell line EndoC- $\beta \mathrm{H} 2-\mathrm{A} 2$, which naturally presents the $\mathrm{PPI}_{15-24}$ - $\mathrm{pHLA}$ epitope recognized by the PD-1 agonist ImmTAAI molecules, as target cells and using $\mathrm{CD}^{+} \mathrm{T}$ cell clones that recognize a distinct pancreatic $\beta$ cell antigen $\mathrm{PPI}_{6-14}$ (RLLPLLALL)-HLA-A2 as effectors (Figure 7A). Cell viability was assessed over time by Incucyte imaging and by labeling target cells with NucLight red to generate EndoC- $\beta \mathrm{H} 2$ Red target cells. Initial cytotoxicity experiments, conducted using a T cell clone with low TCR affinity to PPI ${ }_{6-14}$ pHLA-A2 (clone $4 \mathrm{~b} ; K_{D} \sim 800 \mu \mathrm{M}$; Table 4 ), show that clone $4 \mathrm{~b}$ reduced target $\beta$ cell viability by approximately $50 \%$ at an effector to target $(\mathrm{E}: \mathrm{T})$ ratio of $1: 1$. This decrease in cell viability was almost completely reversed when $\mathrm{T}$ cells were cultured with $\mathrm{PD}$-L1-overexpressing target EndoC- $\beta \mathrm{H} 2$ Red cells (Figure 7, B and C). Likewise, addition of either the H5 or CA949 ImmTAAI molecules also reduced T cellmediated cytotoxicity with picomolar potency (Figure 7B; Supplemental Figure 6, A and B; Supplemental Videos 1 and 2; and Table 5). This effect was dependent on specific ImmTAAI targeting to the EndoC- $\beta$ H2 Red target cells, as PD-1 antibody ImmTAAI molecules containing an irrelevant gp100 TCR showed minimal activity. The PPI ${ }_{15-24}$ TCR alone also had no effect, providing further evidence that the protective activity of the PPI-targeted ImmTAAI molecules was due to PD-1 agonist activity.

Similar results were obtained in a separate experiment using a more potent $\mathrm{T}$ cell clone with a higher-affinity TCR to $\mathrm{PPI}_{6-14}$ pHLA-A2 (clone $12 \mathrm{~b}, K_{D} 90 \mu \mathrm{M}$; Table 4). Clone $12 \mathrm{~b}$ T cells required 100 -fold less target $\mathrm{PPI}_{6-14}$ peptide and a lower E:T ratio than clone $4 \mathrm{~b}$ to achieve a similar level of target cell killing (Figure 7D). Again, the H5 ImmTAAI dose-dependently protected EndoC- $\beta \mathrm{H} 2 \mathrm{Red}$ cells from clone $12 \mathrm{~b}$ T cell-mediated killing with picomolar potency (Figure 7D; Supplemental Figure $6 \mathrm{D})$, increasing cell viability by approximately $50 \%$ above the level observed with maximum cell killing.

Cell culture supernatants from both coculture assays were also assessed for cytokine production. In addition to protecting the $\beta$ cell line from $\mathrm{CD}^{+} \mathrm{T}$ cell clone killing, target cell-bound bispecifics potently inhibited IFN- $\gamma$ (Figure 7, C and E, and Table 5) and TNF- $\alpha$ production (Supplemental Figure 6C). These data demonstrate that cell-bound PD-1 agonist ImmTAAI molecules potently inhibit $\mathrm{CD}^{+} \mathrm{T}$ cell cytokine secretion and confer protection from autoreactive $\mathrm{CD} 8^{+} \mathrm{T}$ cell-mediated cytotoxicity in vitro.

\section{Discussion}

PD-1 agonist ImmTAAI molecules are TCR bispecifics designed to inhibit $\mathrm{T}$ cell activation, through agonism of the PD-1/PD-L1 pathway but only when the $\mathrm{T}$ cell is in a specific organ or tissue. To maximize their effectiveness, they have been engineered to mimic cellular PD-L1 and engage PD-1 at the target cell-T cell interface. Here, we show that cell surface presentation of these PD-1 agonists (via their TCR domain), whether based on PD-L1 itself or potent agonistic antibodies, is essential to their activity, as only target cell-bound ImmTAAI molecules were able to inhibit T cell function. Therefore, this study outlines a design strategy to create efficacious synthetic PD-1 agonist molecules that only confer local immune suppression in the tissue under attack, providing an advantage over systemically acting immune modulators.

This study provides proof of principle that target cell presentation is an important component of the ability of PD-L1 to effectively activate the PD-1 pathway $(20,24,25,31)$. The observation that an ImmTAAI constructed with IgV-PD-L1 was more potent and effective than a flPD-L1 ImmTAAI suggests that the size and orientation of cell-targeted PD-1 agonists is also important for optimal PD-1 activity (32-34). These data are consistent with a previous report, in which lengthening the extracellular domain of PD-1 with additional Ig domains led to incremental reductions in both PD-1 colocalization to TCR microclusters and inhibition of IL-2 production (23).

Antibody-based ImmTAAI molecules were also able to recapitulate the mechanism of PD-L1-mediated PD-1 activation by localizing at the target cell-T cell interface and inhibiting proximal TCR signaling events. However, they were more potent and efficacious than their PD-L1-based counterparts in both 
A

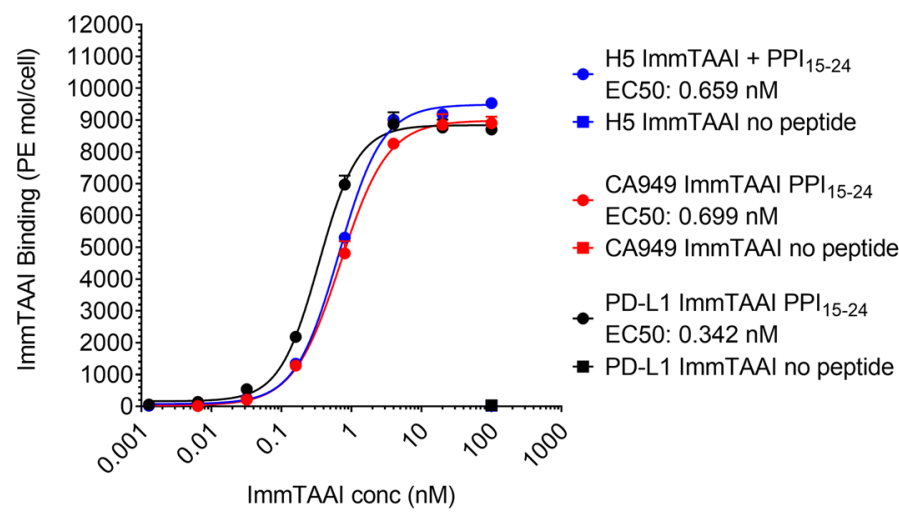

B

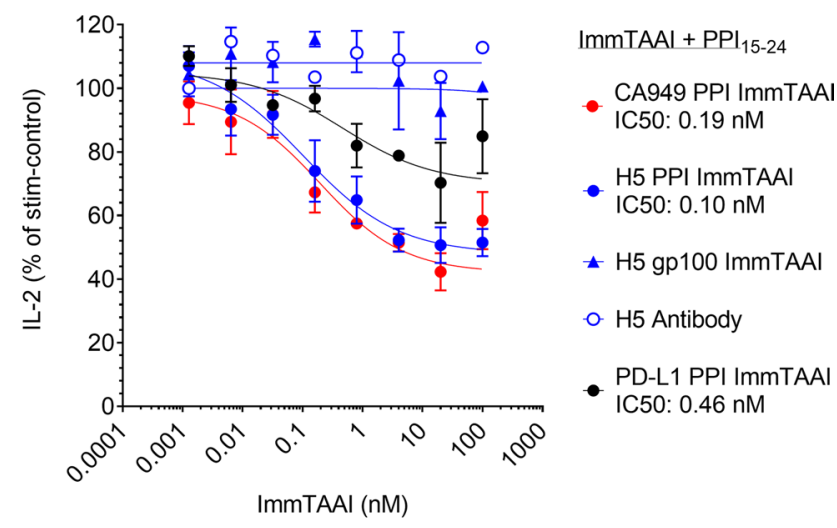

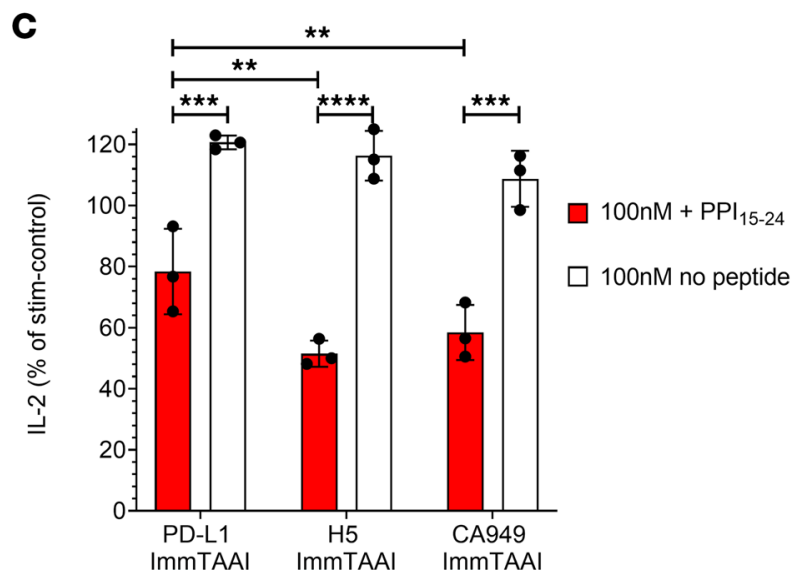

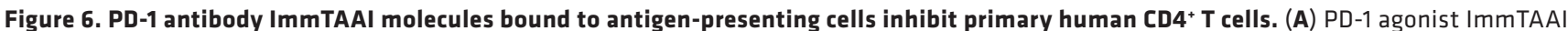
binding to Raji-A2 target cells was analyzed by flow cytometry ( $n=2$ and representative of 3 independent experiments). (B and C) PD- 1 agonist ImmTAAI titrations were preincubated for 1 hour with PPI $_{15-24}$ peptide-pulsed or nonpulsed SEB-loaded Raji-A2 cells. Preactivated T cells were added to the Raji-A2 cells and incubated for 48 hours. Supernatants were collected and IL-2 levels measured by ELISA. Dose response curves plotted to obtain IC $\mathrm{I}_{50}$ values and relative inhibition of IL-2 release at $100 \mathrm{nM} \mathrm{ImmTAAI}$ was plotted for pulsed $\left(+\mathrm{PPI}_{15-24}\right)$ versus nonpulsed target cells $(n=$ 3 and representative of 3 independent experiments). Data are plotted as mean \pm SD and were compared by 2-way ANOVA with repeated measures and Tukey's or Sidak's multiple-comparison test. ${ }^{* *} P \leq 0.01,{ }^{* * *} P \leq 0.001,{ }^{* * * *} P \leq 0.0001$.

reporter and functional assays. Although the mechanisms responsible for this are not fully understood, it is possible that the nanomolar binding affinity of the PD-1 antibodies used in our ImmTAAI molecules influences the strength of PD-1 agonism. The natural affinity of PD-L1 for PD-1 is in the low micromolar range $(35,36)$, and effective $\mathrm{PD}-1-$ mediated suppression of $\mathrm{T}$ cell responses appears to require high levels of PD-1 and PD-L1 expression together with high densities of PD-1-PD-L1 complexes at the T cell-target cell interface $(31,37)$. Such high-order interactions are likely to be necessary to promote PD-1 signaling by providing stability to the PD-1-PD-L1 complexes through increased avidity. The strong affinity of the PD-1 antibodies used in our studies could lead to more stable PD-1 antibody-PD-1 interactions and initiate signaling at a lower receptor occupancy.

Antibody-based ImmTAAI molecules were not only more potent, but also provide additional advantages over PD-L1-based agonists. First, they do not affect the binding of cellular PD-L1 to $\mathrm{PD}-1$ on an interacting $\mathrm{T}$ cell. This feature is not only crucial with regard to lack of interference with physiological tolerance signaling - e.g., in the intestine or placenta - but is of particular relevance in the context of autoimmunity and chronic inflammation, as PD-L1 expression is stimulated by inflammatory cytokines and upregulation of PD-L1 has been observed in certain autoimmune diseases, such as type 1 diabetes (38). Antibody-based ImmTAAI molecules were also additive with cellular PD-L1 in triggering PD-1 activity on T cells, suggesting that our targeted approach could further promote endogenous defence mechanisms during inflammation. Furthermore, the antibody-based ImmTAAI 
Table 3. Raji-A2: primary human T cell IL-2 assay data summary.

\begin{tabular}{|c|c|c|}
\hline Test molecule & Max inhib \% (SD, n) & IC50 nM (SD, n) \\
\hline $\begin{array}{l}\text { VHH H5 ImmTAAI } \\
\text { (PPI }_{15-24} \text { TCR) }\end{array}$ & $52.7(9.3,12)$ & $0.103(0.053,12)$ \\
\hline $\begin{array}{l}\text { scFv CA949 ImmTAAI } \\
\text { (PPI }{ }_{15-24} \text { TCR) }\end{array}$ & $52.0(11.4,9)$ & $0.128(0.058,9)$ \\
\hline $\begin{array}{l}\text { IgV-PD-L1 ImmTAAI } \\
\left(\mathrm{PPI}_{15-24} \text { TCR) }\right.\end{array}$ & $22.3(6.8,3)$ & $0.399(0.054,3)$ \\
\hline $\begin{array}{l}\text { VHH H5 ImmTAAI } \\
\text { (gp100 }_{87-95} \text { TCR) }\end{array}$ & $<10(\mathrm{NA}, 3)$ & $>100(\mathrm{NA}, 3)$ \\
\hline $\mathrm{VHH}$ & $<10(N A, 4)$ & $>100(\mathrm{NA}, 4)$ \\
\hline $\begin{array}{l}\text { scFv CA949 } \\
\text { Ab }\end{array}$ & $<10(\mathrm{NA}, 3)$ & $>100(\mathrm{NA}, 3)$ \\
\hline $\mathrm{PPI}_{15-24} \mathrm{TCR}$ & $<10(N A, 5)$ & $>100(N A, 5)$ \\
\hline
\end{tabular}

molecules do not act as PD-L1-PD-1 antagonists in solution, something that has been suggested for soluble PD-L1 in autoimmune disease $(39,40)$.

Recent studies have shown that there are additional levels of complexity in the regulation of PD-L1-PD-1 signaling in T cells. PD-L1 is able to bind to CD80 in cis on APCs, and this interaction prevents PD-L1-PD-1 binding $(20,41-43)$. PD-L1 can also bind to PD-1 in cis on both APCs and T cells, which either neutralizes or promotes PD-1 signaling in T cells, respectively $(44,45)$. While all of these mechanisms may influence the ability of a PD-L1-based bispecific to promote PD-1 signaling in autoimmune settings, PD-1 antibody-based ImmTAAI molecules can operate as tissue-targeted PD-1 agonists irrespective of CD80 or PD-L1 status.

There is currently some controversy regarding the relative roles of the TCR and CD28 signaling pathways in PD-1-mediated inhibition of $\mathrm{T}$ cell function, with some studies reporting a requirement for CD28 for effective PD-1-mediated T cell inhibition and others indicating that CD28 signaling reduces the ability of PD-1 to suppress T cell function $(24,46-48)$. Despite these disparities, cell-targeted PD-1 antibody ImmTAAI molecules inhibit primary human $\mathrm{T}$ cells, irrespective of the presence or absence of CD28 costimulation. While our research has focused on the ability to trigger the PD- 1 pathway in $\mathrm{CD} 4^{+}$and $\mathrm{CD}^{+}$effector $\mathrm{T}$ cells, it is important to note that PD-1 is expressed on various $\mathrm{T}$ cell subsets and other immune cells, including NK cells, B cells , and macrophages, so it will be important to assess the scope of PD-1 agonists to regulate these different cell types (49-56).

Studies using primary human $\mathrm{T}$ cells allowed the investigation of PD-1 agonist ImmTAAI activity in more physiological settings. Since both the PD-1 agonist and the TCR targeting domain of our molecules are human specific, we did not attempt to use a murine in vivo model (e.g., NOD mice); instead, we generated several in vitro systems to study the effect of PD-1 agonist ImmTAAI molecules on human primary $\mathrm{CD}^{+}$and $\mathrm{CD} 8^{+} \mathrm{T}$ cell function. The Raji-A2 SEB T cell assay models the activation of an antigen-experienced $\mathrm{T}$ cell population, predominantly $\mathrm{CD} 4^{+} \mathrm{PD}-1^{+} \mathrm{T}$ cells, by APCs while the killing studies with insulin-specific $\mathrm{CD} 8^{+} \mathrm{T}$ cell clones model the recognition of self-peptide HLA on pancreatic islet $\beta$ cells by autoreactive $\mathrm{CD} 8^{+} \mathrm{T}$ cells and ensuing $\beta$ cell destruction that occurs in type 1 diabetes. In both models, ImmTAAI molecules were able to potently and efficaciously inhibit $\mathrm{T}$ cell function.

While our studies provide proof of principle for the inhibitory activity of ImmTAAI molecules, further experiments are warranted to understand the scope of these bispecifics more fully. For example, models of APC-mediated activation of autoreactive or allergen-specific CD4 ${ }^{+} \mathrm{T}$ cells may more closely reflect the pathological processes in diseases where these cells predominate. Similarly, $\mathrm{CD} 4^{+}$and $\mathrm{CD} 8^{+}$ $\mathrm{T}$ cell models incorporating several different $\mathrm{T}$ cell clones with distinct specificities could be used to test the ability of PD-1 agonist ImmTAAI molecules to simultaneously suppress multiple T cell reactivities against different antigens on APCs or target cells. Another important question is whether ImmTAAI molecules induce any long-lasting effects on the phenotype and function of $\mathrm{T}$ cells, as such prolonged changes 
A

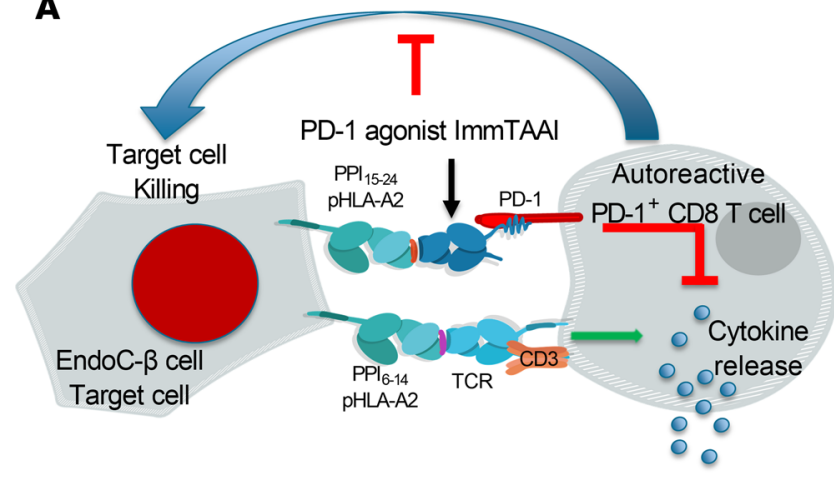

EndoC $-\beta$ cells $+\mathrm{T}$ cell clone + Test agent:

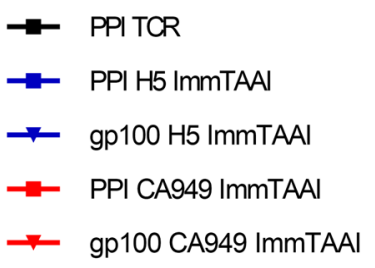

Cell controls:

- EndoC- $\beta$ cells

- EndoC $-\beta$ cells $+\mathrm{T}$ cell clone

- EndoC- $\beta-P D-L 1+T$ cell clone

- EndoC- $\beta-P D-L 1+T$ cell clone + anti-PD-L1

- T cell clone alone
B

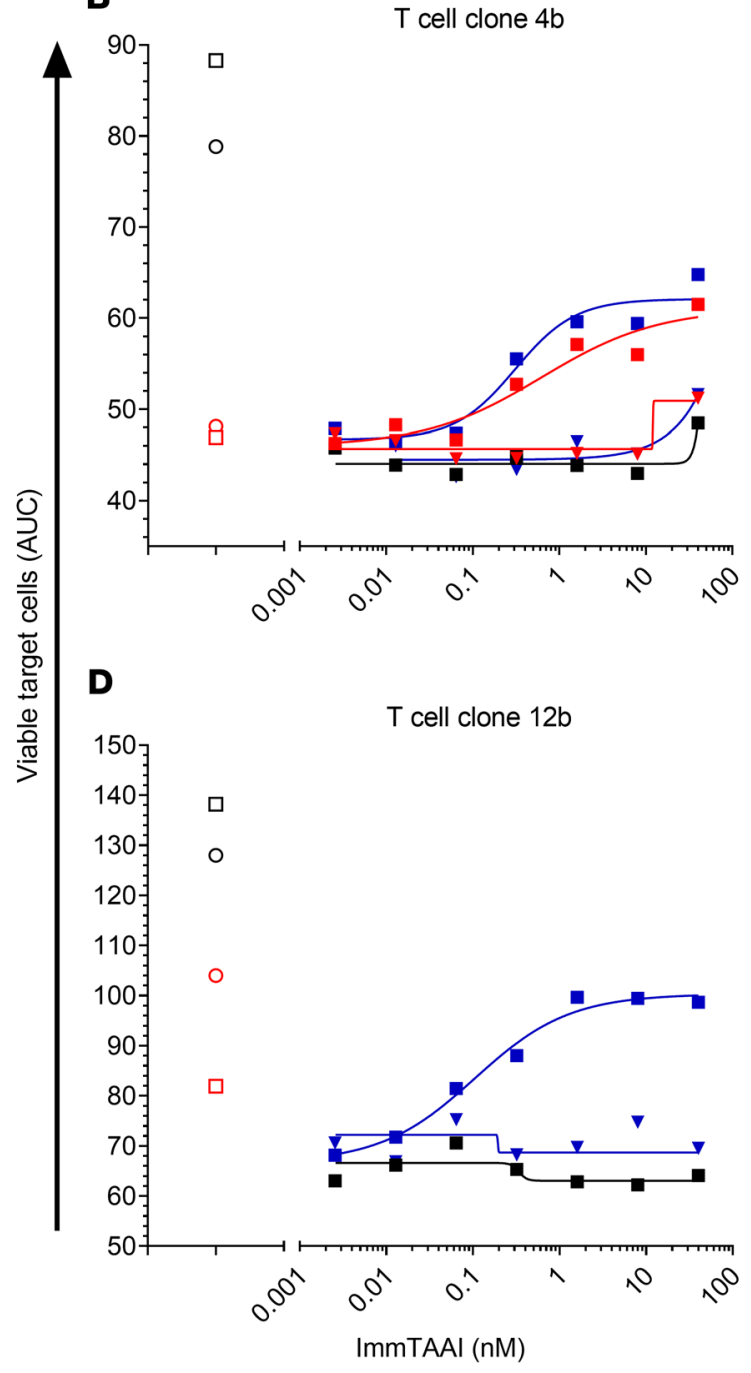

C

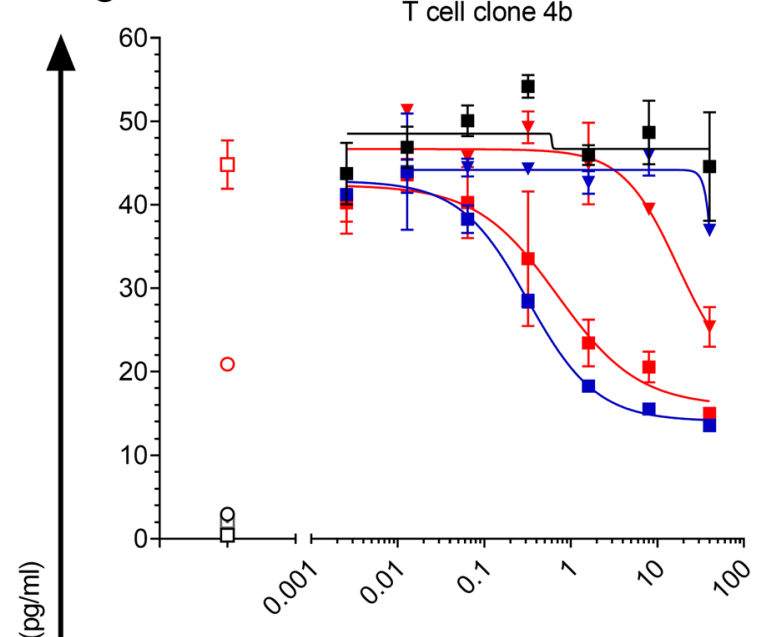

E

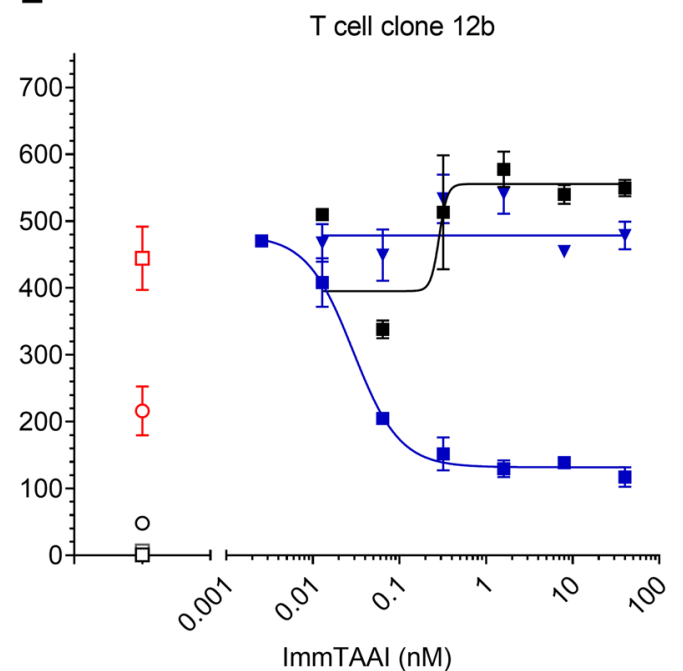

Figure 7. PD-1 antibody ImmTAAI molecules inhibit autoreactive human CD8 ${ }^{+} \mathrm{T}$ cells and protect target cells from $\mathrm{T}$ cell killing. (A) Schematic of the EndoC- $\beta$ Red cell-CD8 ${ }^{+}$T cell clone killing assay. (B and D) PPI ${ }_{6-14}-\mathrm{HLA}$-A2-specific autoreactive T cell clones $4 \mathrm{~b}$ (B) or $12 \mathrm{~b}$ (D) were added to EndoC- $\beta$ Red cells in the presence of PPI antibody or gp100 PD-1 antibody ImmTAAI titrations or TCR only controls. PD-L1-transduced EndoC- $\beta$ Red target cells with or without anti-PD-L1 blocking antibody were used as additional controls. For each sample EndoC- $\beta$ Red target cell number, relative to cells at $(t=0)$ was measured over time by imaging and growth curves generated. AUC was calculated for each curve and $E C_{50}$ data generated by plotting AUC over ImmTAAI concentration (representative data from 3 independent experiments). (C and E) Supernatants were collected from

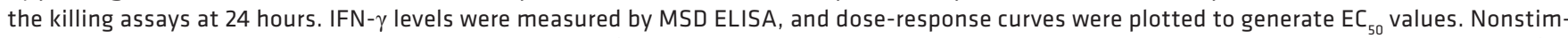
ulated T cells alone were assessed as additional controls ( $n=2$, data are plotted as mean \pm SD and representative of 3 independent experiments). 
Table 4. Characteristics of the $\mathrm{PPI}_{6-14}-\mathrm{HLA}-\mathrm{A2}$-specific T cell clones.

\begin{tabular}{lcc}
\hline CD8 $^{+}$T cell clone & 4b PD-1 $^{+}$ & 12b $_{\text {PD-1 }} \mathbf{1}^{+}$ \\
(PPI $_{6-14}$-HLA-A2) & & \\
TCR affinity $\left(K_{D^{\prime}}, \mu \mathrm{M}\right)$ & $\sim 800$ & 90 \\
$\mathrm{PPI}_{6-14}$ pulse $(\mu \mathrm{M})$ & 1 & 0.01 \\
Effector/ Target cell ratio & $1: 1$ & $0.5: 1$ \\
\hline
\end{tabular}

could impact significantly on pharmacodynamics and efficacy. We therefore plan to investigate the longterm effects of our bispecifics on $\mathrm{T}$ cell function by looking at markers of anergy, exhaustion, and death.

In summary, we have shown that binding a PD-1 agonist to the surface of target cells is a highly effective way to activate the PD-1 receptor on interacting $\mathrm{T}$ cells and achieve immune suppression. Potent PD-1 antibody ImmTAAI molecules closely mimic the mechanism of action of endogenously expressed PD-L1 in their localization to the target cell-T cell interface, inhibition of proximal TCR signaling events, and ultimately suppression of $\mathrm{T}$ cell function. Crucially, in soluble form, the PD-1 ImmTAAI molecules are inactive; they neither activate PD-1 on T cells nor block PD-L1-PD-1 signaling, thus avoiding both systemic immunosuppression and the risk of immune-related adverse events. Our studies highlight a path forward to generate more effective, potent PD-1 agonists that can inhibit T cell function, are biologically inactive in solution, and have scope to treat autoimmune diseases, an area of high unmet need, where effective and safe new therapies are urgently needed.

\section{Methods}

Human PBMC and cell lines. PBMC used in this study were obtained from healthy volunteers (Immunocore employees). The Jurkat NFAT luciferase T cell line was purchased from Promega and maintained in RPMI-1640 supplemented with 10\% heat-inactivated FBS, 2 mM L-glutamine, 0.1 mM MEM nonessential amino acids, $1 \mathrm{mM}$ sodium pyruvate, pen/strep (50 U/mL penicillin and $50 \mu \mathrm{g} / \mathrm{mL}$ strep), and $200 \mu \mathrm{g} / \mathrm{mL}$ hygromycin B. The Raji B cell lymphoma line was purchased from ATCC and maintained in R10 growth media (RPMI-1640 supplemented with 10\% heat-inactivated FBS, 2 mM L-glutamine, and pen/strep). HEK-293T cells were purchased from ATCC and maintained in DMEM (4.5 g/L glucose) supplemented with $10 \%$ heat-inactivated FBS and $2 \mathrm{mM}$ L-glutamine. The immortalized human pancreatic $\beta$ cell lines, ECN90 and EndoC- $\beta$ H2, were purchased from Univercell Biosolutions and maintained in Optiß3 and Opti $\beta 1$ media, respectively, in tissue culture vessels precoated with $\beta$-coat (Univercell Biosolutions).

All standard media (RPMI-1640, DMEM), FBS, glutamine, MEM nonessential amino acids, sodium pyruvate, pen/strep, PHA, and PBS were purchased from Thermo Fisher Scientific. Hygromycin B was purchased from Invitrogen. Dithiothreitol was from Sigma-Aldrich.

TCR engineering. TCRs targeting PPI $_{15-24}$ (HLA-A*02:01, ALWGPDPAAA) or gp $100_{280-288}$ (HLA-A*02:01, YLEPGPVTA) peptide HLAs were isolated either from in-house phage libraries using standard phage-display methods (57) or from $\mathrm{T}$ cell cloning using human donors. TCRs were then affinity enhanced through molecular evolution using phage display as previously described (58).

Agonist PD-1 antibody generation. PD-1-specific antibodies were generated by immunization of 1lamas with recombinant human PD-1-His protein (Acro Biosystems, PD1-H5221) and after 2-3 rounds of phage-display panning on immobilized recombinant human PD-1-Fc protein (Acro Biosystems, PD1H5257), using adapted methods from literature (59).

ImmTAAI production. ImmTAAI molecules were generated by fusing the affinity-matured TCRs to the desired PD-1 agonist effector moiety. Effector moieties included truncated PD-L1 comprising the IgV domain (residues 19-134), PD-L1 full extracellular domain, an scFv PD-1 agonist antibody (CA949), and VHH PD-1 agonist antibodies. The resulting constructs were produced either by expression as inclusion bodies in E. coli followed by refolding and purification as described previously (60), or soluble expression in mammalian cells (Expi-CHO-S, Thermo Fisher Scientific) followed by purification using immobilized metal affinity chromatography and size exclusion chromatography.

Lentivirus production and transduction. Lentiviral vectors containing PDCD1 (PD-1) and CD274 (PDL1) were obtained from OriGene (RC210364L1 and RC213071L1, respectively). NucLight red (IncuCyte 
Table 5. CD8+ $T$ cell clone-EndoC- $\beta$ Red cell killing and cytokine assay data summary

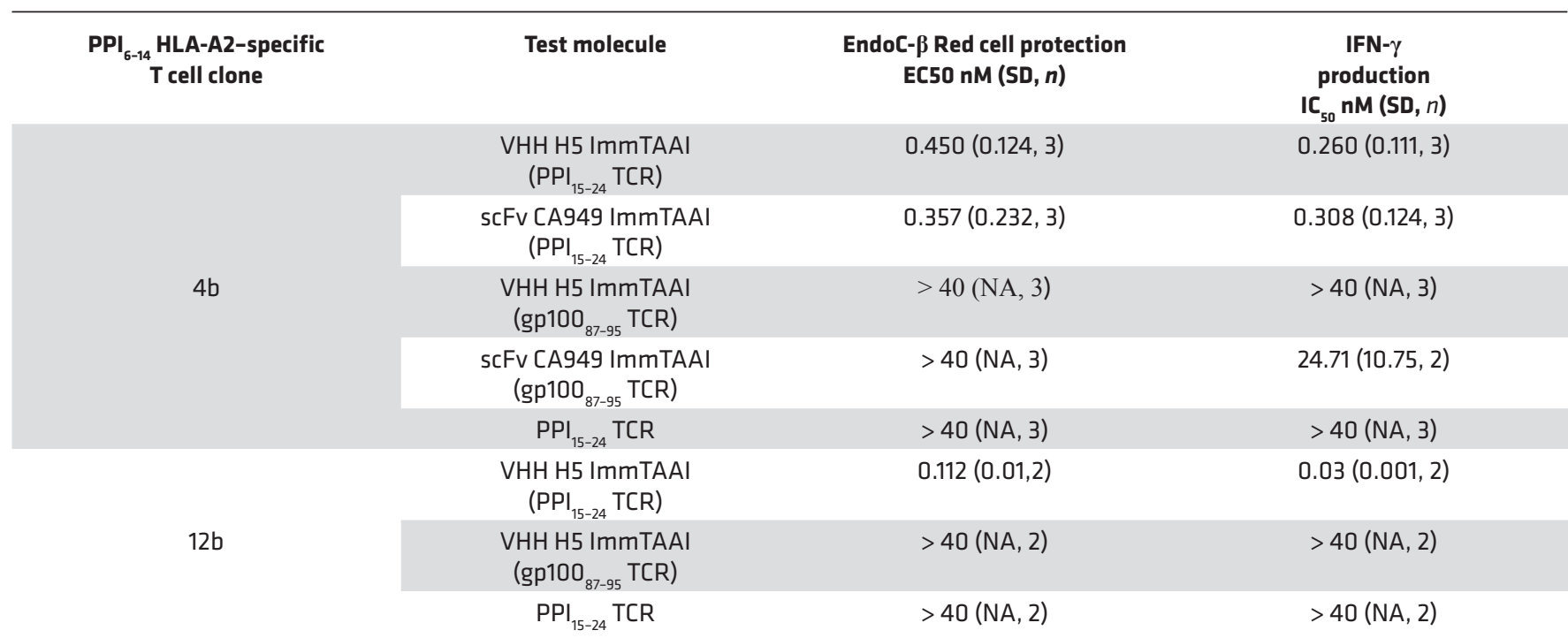

For the killing assays, growth curves were generated over 60-80 hours, and AUC values calculated. $\mathrm{EC}_{50}$ values for EndoC- $\beta$ cell protection were calculated by fitting plots of AUC values versus ImmTAAI concentration. NA, not applicable; O/E, overexpressing; scFv, single-chain fragment variable; Ab, antibody.

NucLight Red Lentivirus Reagent) was obtained from Essen Bioscience. Lentiviral vectors for HLA-A2 $\beta 2$-microglobulin and the Mel5 TCR were produced in-house. Lentiviruses were packaged by transfection of lentiviral vectors with packaging plasmids (pMD2.g, pMDLg/p RRE, and pRSV.REV, in-house) into HEK293T cells with TurboFect Transfection Reagent (Thermo Fisher Scientific). Lentiviral particles were collected 48 hours after transfection, filtered through a $0.45 \mu \mathrm{m}$ filter (Nalgene), and concentrated by centrifugation at $10,000 \mathrm{~g}$ for 16 hours at $4^{\circ} \mathrm{C}$. Lentivirus pellets were resuspended in growth media and stored at $-80^{\circ} \mathrm{C}$ prior to use. For transduction of cell lines, lentivirus was added to $1 \times 10^{6}$ of exponentially growing cells in wells of a 24-well cell culture plate. After 48-72 hours, transduced cells were harvested, assessed for expression of transduced genes by flow cytometry, and expanded into cell culture flasks. Where necessary, cells were sorted by FACS or enriched using anti-PE beads (Miltenyi Biotec), to obtain populations of cells $>95 \%$ positive for the transduced gene product of interest. For transduction of $\mathrm{T}$ cell clones with PD-1 lentivirus, $1 \times 10^{6} \mathrm{~T}$ cell clones were stimulated with a mix of irradiated PBMC from 4 donors in the presence of $2 \mu \mathrm{g} / \mathrm{mL}$ PHA and $100 \mathrm{U} / \mathrm{mL}$ recombinant human IL-2 (Peprotech) for 24 hours. Lentivirus was added to the coculture and incubated for several days. Transduction efficiency was evaluated by flow cytometry. PD-1+ $\mathrm{T}$ cell clones were sorted with (Sony Biotechnology), and cells were reexpanded with feeders in the presence of PHA and recombinant IL-2 at $100 \mathrm{U} / \mathrm{mL}$. T cell clones were used in the killing assay after 20 days.

Jurkat NFAT luciferase PD-1 reporter assays. HEK293T: Jurkat NFL PD-1 reporter assay, HEK293T-A2 target cells, were washed and resuspended in D10 media without antibiotics (DMEM, 10\% FBS) and plated into white 96 -well cell culture plates $\left(50,000\right.$ cells/well) at $37^{\circ} \mathrm{C}, 5 \% \mathrm{CO}_{2}$ for 24 hours. The HEK293T cells were transfected with $100 \mathrm{ng}$ membrane-bound anti-CD3 antibody plasmid or with $100 \mathrm{ng}$ membrane-bound anti-CD3 antibody + PD-L1 plasmid (BPS Bioscience) and $0.3 \mu \mathrm{L}$ Lipofectamine 2000 (Invitrogen) per well, using Opti-MEM I (Thermo Fisher Scientific) as diluent. After 24 hours, the cells were either pulsed by adding D10 media containing $20 \mu \mathrm{M}$ PPI peptide (ALWGPDPAAA) or mock-treated with D10 media alone. After incubating for 2 hours at $37^{\circ} \mathrm{C}, 5 \% \mathrm{CO}_{2}$, media was removed from each well, and assay buffer alone (RPMI-1640, supplemented with 10\% heat-inactivated FBS and $2 \mathrm{mM} \mathrm{L}$-glutamine) or assay buffer containing titrations of PD-1 agonist ImmTAAI molecules or PD-L1 Fc (R\&D Systems) was added to each well. The assay was initiated by adding 50,000 Jurkat NFL PD-1 effector cells in assay buffer and incubating for $16-20$ hours at $37^{\circ} \mathrm{C}, 5 \% \mathrm{CO}_{2}$.

ECN90-Jurkat NFL Me15 PD-1 reporter assay - ECN90 or ECN90-PD-L1 cells were harvested and plated at 50,000 cells/well in Optiß3 media, into the inner 60 wells of a white 96 -well cell culture plate 
that had been precoated with $\beta$-coat (Univercell Biosolutions). After incubating at $37^{\circ} \mathrm{C}, 5 \% \mathrm{CO}_{2}$ for $16-20$ hours, media was removed and assay buffer containing $10 \mu \mathrm{M}$ Melan-A peptide (ELAGIGILTV) was added. After pulsing for 2 hours at $37^{\circ} \mathrm{C}, 5 \% \mathrm{CO}_{2}$, assay buffer alone or assay buffer containing titrations of PD-1 agonist ImmTAAI molecules was added to each well. The assay was initiated by immediately adding 50,000 Jurkat NFL Mel5 PD-1 effector cells and incubating for $16-20$ hours at $37^{\circ} \mathrm{C}, 5 \% \mathrm{CO}_{2}$.

Both reporter assays were developed by adding BioGlo reagent (Promega), and NFAT activity was determined by measuring luciferase luminescence on a plate reader (Clariostar, BMG Labtech). NFAT activity was normalized against TCR-stimulated controls and ImmTAAI dose-response data were analyzed in Prism (GraphPad) using a 4 parameter, nonlinear least squares fit to determine $\mathrm{IC}_{50}$ values. For the HEK293T: Jurkat NFL PD-1 reporter assays, relative NFAT activity at $100 \mathrm{nM}$ ImmTAAI for pulsed $\left(+\mathrm{PPI}_{15-24}\right.$ ) versus nonpulsed (no peptide) target cells was also plotted to highlight the targeting-dependency of PD-1 agonist ImmTAAI-mediated inhibition.

Primary human T cell IL-2 assay. Primary human T cells were isolated from PBMCs using a pan T cell isolation kit (Miltenyi Biotec) and resuspended in R10. The T cells were preactivated by incubating with irradiated Raji A2 cells, preloaded with $1 \mu \mathrm{g} / \mathrm{mL}$ SEB (MilliporeSigma), using $1 \times 10^{6} \mathrm{~T}$ cells to $1 \times 10^{6}$ Raji cells per well of 24-well tissue culture plates. Preactivation plates were incubated for 10 days at $37^{\circ} \mathrm{C}, 5 \% \mathrm{CO}_{2}$ and IL-2 (50 $\mathrm{U} / \mathrm{mL}$ ) added at days 3 and 7 . After preactivation, the expanded $\mathrm{T}$ cells were predominantly $\mathrm{CD} 4^{+} \mathrm{T}$ cells and typically $60 \%-70 \% \mathrm{PD}-1^{+}$. The T cells were washed 3 times in R10 to remove IL-2 and dead cell debris, and they were resuspended in fresh R10. For restimulation of preactivated T cells in the presence of target cellbound or soluble PD-1 agonist ImmTAAI molecules, Raji-A2 cells were pulsed, or not, with $20 \mu \mathrm{M}$ PPI ${ }_{15-24}$ peptide at $2 \times 10^{6} \mathrm{cell} / \mathrm{mL}$ in R10 for 2 hours at $37^{\circ} \mathrm{C}, 5 \% \mathrm{CO}_{2}$. Raji-A2 and Raji-A2-PD-L1 cells were then loaded with $31.6 \mathrm{ng} / \mathrm{mL}$ SEB for 1 hour at $37^{\circ} \mathrm{C}, 5 \% \mathrm{CO}_{2}$, and irradiated with 33 Gy. Raji-A2 and Raji-A2PD-L1 cells were plated into the inner 60 wells of flat-bottomed 96-well culture plates at 100,000 cells/well. R10 alone or R10 containing PD-1 agonist ImmTAAI dilutions was added to PPI pulsed or nonpulsed Raji-A2 cells. For PD-L1 inhibition controls, R10 alone or R10 containing a PD-L1 blocking antibody at a final concentration of $12.5 \mu \mathrm{g} / \mathrm{mL}$ (BioLegend, catalog 329716, clone 29E.2A3) was added to Raji-A2-PD-L1 cells. After 1 hour preincubation, washed preactivated T cells were added to the Raji-A2 cells at 100,000 cells/well and incubated for 48 hours at $37^{\circ} \mathrm{C}, 5 \% \mathrm{CO}_{2}$. Supernatants were collected, and IL-2 levels were measured by ELISA (IL2 Ready-SET-Go! ELISA, Invitrogen). IL-2 release was normalized against SEB-stimulated controls, and ImmTAAI dose response data were analyzed in Prism (GraphPad) using a 4 parameter, nonlinear least squares fit to determine $\mathrm{IC}_{50}$ values. Relative IL-2 levels at $100 \mathrm{nM}$ ImmTAAI for pulsed (+ PPI ${ }_{15-24}$ ) versus nonpulsed (no peptide) target cells were also plotted to highlight the targeting-dependency of PD-1 agonist ImmTAAI-mediated inhibition.

ImmTAAI colocalization studies. H5 PPI ImmTAAI was labeled with AF488 using the CF488A SE Protein Labeling Kit (Biotium) according to the manufacturer's instructions. ECN90 cells were harvested, washed in PBS 10\% FBS, and resuspended at $1 \times 10^{6}$ cells $/ \mathrm{mL}$ in OpitiB3 (Univercell Biosolutions). Cells were pulsed with $2.5 \mu \mathrm{M}$ Melan-A peptide for 2 hours, washed with PBS, and resuspended at $1 \times 10^{6}$ cells/ $\mathrm{mL}$ in PBS $2 \%$ FBS. ECN90 cells were incubated with 100nM AF488-ImmTAAI at room temperature for 30 minutes. Excess ImmTAAI was washed away with PBS 2\% FBS and resuspended in RPMI (no FBS) at $0.8 \times 10^{6}$ cells $/ \mathrm{mL}$ in RPMI. ECN90 cells were added to glass slide chambers (Ibidi) at 80,000 cells/well, incubated at $37^{\circ} \mathrm{C}$ for 10 minutes. Jurkat NFL Mel5 parental and PD-1 cells were resuspended at $0.8 \times 10^{6}$ cells/mL in R10 and added to ECN90 cells at a 1:1 ratio (80,000 Jurkat cells/sample) and incubated for 30 minutes at $37^{\circ} \mathrm{C}$. Cells were fixed for 20 minutes with Fixative BD stabilizing. The reaction was quenched by washing with PBS $2 \%$ FBS. $Z$ stack images were acquired using a confocal microscope, Nikon (Nikon Eclipse Ti2 and Nikon A1 confocal system), and Imaging analysis was performed with Image J (Fiji). For each condition (Jurkat NFL Mel5 PD-1 or Jurkat NFL Mel5 parental cells), the number of ECN90-Jurkat conjugates exhibiting accumulation of AF488-ImmTAAI was counted and plotted as a percentage of the total number of conjugates.

TCR stimulation and Western blot studies. ECN90 and ECN90-PD-L1 cells were harvested by washing twice in PBS and resuspending in nonenzymatic cell dissociation buffer (Thermo Fisher Scientific). ECN90 and ECN90-PD-L1 cells were resuspended at $2 \times 10^{6} / \mathrm{mL}$ in Optiß3 and pulsed, or not, with $10 \mu \mathrm{M}$ Melan-A peptide for 2 hours at $37^{\circ} \mathrm{C}$ on a rotator. After pulsing, cell suspensions were diluted 1:2 with either media alone or media containing $50 \mathrm{nM}$ PD-1 antibody ImmTAAI. ECN90 cells with or without PD-1 antibody ImmTAAI were incubated on a rotator for 2 hours at $37^{\circ} \mathrm{C}$. Unbound PD-1 
antibody ImmTAAI was removed by washing the ECN90 cells twice in RPMI-1640 and resuspended at $20 \times 10^{6} / \mathrm{mL}$ in RPMI alone by removing the required volume of media. During the preparation of the ECN90 target cells, Jurkat NFL Mel5 PD-1 effector cells were washed twice in RPMI-1640, resuspended in RPMI alone at $20 \times 10^{6} \mathrm{cells} / \mathrm{mL}$, and starved at $37^{\circ} \mathrm{C}$ for 4 hours on a rotator. Stimulation time course studies with Melan-A-pulsed target cells and Jurkat Mel5 cells established that maximal TCR-stimulated phosphorylation of the signaling molecules SLP-76, PLC $\gamma$, and ZAP-70 occurred within a 10-minute time frame. TCR stimulations were initiated by adding $250 \mu \mathrm{L}$ Jurkat NFL Mel5 PD-1 cells at $20 \times 10^{6} / \mathrm{mL}$ to $250 \mu \mathrm{L}$ ECN90 cells in $1.5 \mathrm{~mL}$ Eppendorf tubes and pelleting at $400 \mathrm{~g}$ for 10 seconds to promote effector cell/target cell contact. Stimulations were conducted over a 10-minute time-course and stopped by spinning down at 17,000 $\mathrm{g}$ in a microfuge for 12 seconds, removing the supernatant and adding $250 \mu \mathrm{L}$ NP-40 lysis buffer (Alfa Aesar), supplemented with protease inhibitor cocktail (MilliporeSigma) and phosphatase inhibitor cocktail (PhosSTOP, Roche). Cells were lysed on ice for 20 minutes, and nonsoluble material was pelleted by spinning at $20,000 \mathrm{~g}$ in a microfuge at $4^{\circ} \mathrm{C}$ for 10 minutes. Protein levels of clarified cell lysates was determined by BCA assay (Thermo Fisher Scientific), and whole cell lysates were prepared by adding the clarified lysates to $4 \times$ Laemmli sample buffer (Bio-Rad) containing $200 \mathrm{mM}$ dithiothreitol. Samples were denatured at $95^{\circ} \mathrm{C}$ for 5 minutes and then stored at $-80^{\circ} \mathrm{C}$ for subsequent Western blotting. Samples were run on denaturing 4\%-20\% SDS-PAGE gels (Bio-Rad) at $50 \mu \mathrm{g}$ protein/sample and transferred to PVDF membranes (Bio-Rad). Membranes were probed with primary antibodies specific for phospho-SLP76 (pY128, BD Pharmingen, catalog 558367, clone J141-668.36.58), phospho-PLC $\gamma$ (pY783, Cell Signaling Technology, catalog 2821), and phospho-ZAP-70 (pY319, Cell Signaling Technology, catalog 2701) and developed using HRP-conjugated secondary antibodies (Amersham, GE Healthcare anti-mouse IgG HRP and anti-rabbit IgG HRP, catalogs NA931 and NA934, respectively) and ECL reagent (SuperSignal West Pico Plus ECL substrate, Thermo Fisher Scientific). Membranes were stripped using Restore Western Blot Stripping buffer (Thermo Fisher Scientific, catalogs 4958, 5690 and 2709) and reprobed using antibodies specific for total SLP-76, PLC $\gamma$, and ZAP-70 (Cell Signaling Technology). Chemiluminescence was detected and quantified with a Chemidoc Imager and Image Lab software (Bio-Rad).

Flow cytometry and ImmTAAI-target cell binding studies. Flow cytometric studies of human cell lines and primary $\mathrm{T}$ cells were conducted using the following fluorophore-conjugated antibodies: anti-CD3 (Invitrogen, clone OKT3), anti-CD4 (Invitrogen, clone RPA-T4), anti-CD8 (Invitrogen, clone RPA-T8), anti-PD-1 (BioLegend, clone EH12.2H7), anti-HLA-A2 (BioLegend, clone BB7.2), anti-PD-L1 (BioLegend, clone 29E.2A3), anti-CD80 (BD Pharmingen, clone L307.4), anti-CD86 (BD Pharmingen, clone FUN-1). After staining for dead cells with Live/Dead Fixable Aqua Dead Stain (Invitrogen), cell surface staining was conducted using 1 in 100 dilutions of the antibodies in FACS wash buffer (FWB; PBS, 0.5\% FBS), incubating at $4^{\circ} \mathrm{C}$ for 30 minutes, followed by 2 washes in FWB. PPI ${ }_{15-24}$ HLA-A2-specific binding of FLAG-tagged ImmTAAI molecules to target cells was determined by incubating target cells (with or without prior $\mathrm{PPI}_{15-}$ ${ }_{24}$ peptide pulsing) with ImmTAAI dilutions in growth media at $37^{\circ} \mathrm{C}, 5 \% \mathrm{CO}_{2}$, for 3 hours. Target cells were washed in FWB and stained with an anti-FLAG-PE antibody (BioLegend, catalog 329706, clone L5) as described for the cell surface antibodies. Data were acquired on an IntelliCyt iQue Plus (Sartorius), and estimates of surface molecule/cell were calculated using PE Quantibrite Bead analysis (BD Biosciences). ImmTAAI binding dose response data were analyzed in Prism (GraphPad) using a 4 parameter, nonlinear least squares fit to calculate $\mathrm{EC}_{50}$ values

Generation of $T$ cell clones. T cell clones specific for the pancreatic $\beta$ cell antigen PPI ${ }_{6-14}$ (HLA-A*02:01 RLLPLLALL) were generated as described (61). T cell clone specificity was validated by dextramer staining (made in-house from biotinylated $\mathrm{PPI}_{15-24}$ pHLA-A2 and fluorescently labeled Streptavidin-Dextramer, Immundex ApS) and specific killing of EndoC- $\beta$ H2-A2 Red cells pulsed with RLLPLLALL peptide (Incucyte S3, Sartorius).

T cell killing assay and cytokine analyzes. EndoC- $\beta \mathrm{H} 2$ Red cells were generated by transducing EndoC- $\beta \mathrm{H} 2$ cells with HLA-A2 $\beta 2$-microglobulin and NucLight red (Sartorius). Target cells were plated at $5 \times 10^{4}$ cells per well of a 96-well plate in Optiß3 media, incubated over night at $37^{\circ} \mathrm{C}, 5 \% \mathrm{CO}_{2}$, and then pulsed with $\mathrm{PPI}_{6-14}$ peptide at the indicated concentrations for 2 hours. ImmTAAI molecules and controls were added at different concentrations and incubated for 2 hours. To initiate the assay, T cell clones were washed twice and added to EndoC- $\beta$ H2 Red target cells at $5 \times 10^{4}$ cells per well in T cell cloning media. PD-L1 transduced EndoC- $\beta$ H2 Red target cells with or without anti-PD-L1 blocking antibody were used as additional controls. Cell killing was determined by quantification of EndoC- $\beta \mathrm{H} 2$ Red cell number over time using the IncuCyte 
S3 imaging system (Sartorius). The number of red nucleus-labeled cells at each time point was normalized to the initial number of objects to take into account variation in cell density in the area visualized. The number of events was acquired in 4 images and averaged. Cytokine release was measured by V-PLEX Plus Proinflammatory Panel 1 (human) kit in accordance with the manufacturer's instructions (Meso Scale Diagnostics [MSD]) using culture supernatants from the IncuCyte killing assays at 24 hours after time point. For the cytokine assay, nonstimulated $\mathrm{T}$ cells alone were assessed as additional controls. Cytokine release was normalized against stimulated controls, and ImmTAAI dose response data were analyzed in Prism (Graph$\mathrm{Pad}$ ) using a 4-parameter, nonlinear least squares fit to determine $\mathrm{IC}_{50}$ values.

SPR kinetic analysis. SPR competition binding analysis was performed using a BIAcore $8 \mathrm{~K}$. Briefly, biotinylated PPI peptide HLA-A2 complexes were immobilized on a streptavidin-coated CM5 chip. ImmTAAI molecules were captured onto the chip via the affinity-enhanced PPI $_{15-24}$ TCR-PPI ${ }_{15-24}$ peptide HLA-A2 interaction. Excess PD-1 was passed over the chip $\left(1 \mu \mathrm{M}\right.$ or $10 \times K_{D}$ of each PD-1 antibody), followed by an excess of PD-1 and PD-L1-Fc $(15 \mu \mathrm{M})$ (Supplemental Figure 3A).

Statistics. Statistical analysis was performed using GraphPad Prism. Unless stated otherwise, data are presented as the mean \pm SD. Data were analyzed using 2-way ANOVA with repeated measures by Tukey's or Sidak's multiple-comparison test or by 1-way ANOVA and Dunnett's multiple-comparison test. Four-parameter nonlinear regression analysis was used to fit dose-response curves and calculate $\mathrm{IC}_{50}$ and $\mathrm{EC}_{50}$ values.

Study approval. The Oxford A Research Ethics Committee approved protocol 13/SC/0226 (Immunocore study protocol number IMCres02) was used to obtain written consent for all blood donations and was fully approved by the National Research Ethics Committee South Central.

\section{Author contributions}

APC, GB, JK, LJB, RF, RT, KW, EH, SJH, KP, NMGS, LMW, VG, and CRR performed and analyzed experiments. HG, DEOK, RO, DXO, and CML provided reagents. APC, GB, RF, RT, SH, TMM, and PW designed and interpreted experiments. APC, MLM, and PW prepared the manuscript. All authors critically read the manuscript.

\section{Acknowledgments}

We thank Marco Lepore and Lucy Walker for helpful discussions. David Cole and Chris O'Callaghan critically reviewed the manuscript.

Address correspondence to: Adam P. Curnock or Peter Weber, 92 Park Drive, Milton Park, Abingdon, United Kingdom. Phone: 44.1235.438600. Email: adam.curnock@immunocore.com (APC); Email: peter. weber@immunocore.com (PW).

JK's present address is: GammaDelta Therapeutics, White City Place, London W12 7FQ, United Kingdom. RT's present address is: Evox Therapeutics, Littlemore, Oxford OX4 4HG, United Kingdom. SJH's present address is: Antigen Presentation Research Group, Department of Infectious Disease, Imperial College London, United Kingdom. EOK's present address is: DJS antibodies, Cherwell Innovation Centre, Upper Heyford, OX25 5HD, United Kingdom. CML's present address is: oNKo-Innate Pty. Ltd, Moonee Ponds VIC 3039, Australia. CRR's present address is: Biopharm Discovery, GlaxoSmithKline, Stevenage SG1 2NY, United Kingdom.

1. Okazaki T, et al. A rheostat for immune responses: the unique properties of PD-1 and their advantages for clinical application. Nat Immunol. 2013;14(12):1212-1218.

2. Sharpe AH, Pauken KE. The diverse functions of the PD1 inhibitory pathway. Nat Rev Immunol. 2018;18(3):153-167.

3. Patsoukis N, et al. Revisiting the PD-1 pathway. Sci Adv. 2020;6(38):eabd2712.

4. Freeman GJ, et al. Engagement of the PD-1 immunoinhibitory receptor by a novel B7 family member leads to negative regulation of lymphocyte activation. J Exp Med. 2000;192(7):1027-1034.

5. Latchman Y, et al. PD-L2 is a second ligand for PD-1 and inhibits T cell activation. Nat Immunol. 2001;2(3):261-268.

6. Taube JM, et al. Colocalization of inflammatory response with B7-h1 expression in human melanocytic lesions supports an adaptive resistance mechanism of immune escape. Sci Transl Med. 2012;4(127):127ra37.

7. Scandiuzzi L, et al. Tissue-expressed B7-H1 critically controls intestinal inflammation. Cell Rep. 2014;6(4):625-632

8. Topalian SL, et al. Immune checkpoint blockade: a common denominator approach to cancer therapy. Cancer Cell. 
2015;27(4):450-461

9. Qian C, et al. Association of PD-1 and PD-L1 genetic polymorphyisms with type 1 diabetes susceptibility. J Diabetes Res. 2018;2018:1614683

10. Yang M, et al. The programmed cell death 1 gene polymorphisms (PD 1.3 G/A, PD 1.5 C/T and PD 1.9 C/T) and susceptibility to ankylosing spondylitis: a meta-analysis. J Orthop Sci. 2015;20(1):55-63.

11. Huang $\mathrm{CH}$, et al. Effects of genetic polymorphisms of programmed cell death 1 and its ligands on the development of ankylosing spondylitis. Rheumatology (Oxford). 2011;50(10):1809-1813.

12. Kroner A, et al. A PD-1 polymorphism is associated with disease progression in multiple sclerosis. Ann Neurol. 2005;58(1):50-57.

13. Prokunina L, et al. A regulatory polymorphism in PDCD1 is associated with susceptibility to systemic lupus erythematosus in humans. Nat Genet. 2002;32(4):666-669.

14. Ben Nasr M, et al. PD-L1 genetic overexpression or pharmacological restoration in hematopoietic stem and progenitor cells reverses autoimmune diabetes. Sci Transl Med. 2017;9(416):eaam7543.

15. Beswick EJ, et al. Expression of programmed death-ligand 1 by human colonic CD $90^{+}$stromal cells differs between ulcerative colitis and crohn's disease and determines their capacity to suppress Th1 cells. Front Immunol. 2018;9:1125.

16. Ekman I, et al. Circulating CXCR5(-)PD-1(hi) peripheral T helper cells are associated with progression to type 1 diabetes. Diabe tologia. 2019;62(9):1681-1688.

17. Awad SS, et al. Expression of immune checkpoints in active nonsegmental vitiligo: a pilot study. Int J Dermatol. 2020;59(8):982-988.

18. Tocut M, et al. Autoimmune phenomena and disease in cancer patients treated with immune checkpoint inhibitors. Autoimmun Rev. 2018;17(6):610-616.

19. Leonardi GC, et al. Safety of programmed death-1 pathway inhibitors among patients with non-small-cell lung cancer and preexisting autoimmune disorders. J Clin Oncol. 2018;36(19):1905-1912.

20. Zhao Y, et al. PD-L1:CD80 cis-heterodimer triggers the co-stimulatory receptor CD28 while repressing the inhibitory PD-1 and CTLA-4 pathways. Immunity. 2019;51(6):1059-1073.

21. Chemnitz JM, et al. SHP-1 and SHP-2 associate with immunoreceptor tyrosine-based switch motif of programmed death 1 upon primary human T cell stimulation, but only receptor ligation prevents T cell activation. J Immunol. 2004;173(2):945-954

22. Sheppard KA, et al. PD-1 inhibits T-cell receptor induced phosphorylation of the ZAP-70/CD3zeta signalosome and downstream signaling to PKCtheta. FEBS Lett. 2004;574(1-3):37-41.

23. Yokosuka T, et al. Programmed cell death 1 forms negative costimulatory microclusters that directly inhibit T cell receptor signaling by recruiting phosphatase SHP2. J Exp Med. 2012;209(6):1201-1217.

24. Hui E, et al. T cell costimulatory receptor CD28 is a primary target for PD-1-mediated inhibition. Science. 2017;355(6332):1428-1433.

25. Celis-Gutierrez J, et al. Quantitative interactomics in primary T cells provides a rationale for concomitant PD-1 and BTLA Coinhibitor blockade in cancer immunotherapy. Cell Rep. 2019;27(11):3315-3330.

26. Marasco M, et al. Molecular mechanism of SHP2 activation by PD-1 stimulation. Sci Adv. 2020;6(5):eaay4458.

27. Patsoukis N, et al. Interaction of SHP-2 SH2 domains with PD-1 ITSM induces PD-1 dimerization and SHP-2 activation. Commun Biol. 2020;3(1):128

28. Skowera A, et al. CTLs are targeted to kill beta cells in patients with type 1 diabetes through recognition of a glucose-regulated preproinsulin epitope. J Clin Invest. 2008;118(10):3390-3402.

29. Tyson KL inventors; UCB Pharma, S.A. Biological products: humanised agonistic anti-PD-1 antibodies. WO patent WO2011110621A1. September 15, 2011

30. Cox AL, et al. Identification of a peptide recognized by five melanoma-specific human cytotoxic T cell lines. Science. 1994;264(5159):716-719.

31. Wei F, et al. Strength of PD-1 signaling differentially affects T-cell effector functions. Proc Natl Acad Sci U S A. 2013;110(27):E2480-E2489.

32. Choudhuri K, et al. T-cell receptor triggering is critically dependent on the dimensions of its peptide-MHC ligand. Nature 2005;436(7050):578-582.

33. Davis SJ, van der Merwe PA. The kinetic-segregation model: TCR triggering and beyond. Nat Immunol. 2006;7(8):803-809.

34. Paluch C, et al. Immune checkpoints as therapeutic targets in autoimmunity. Front Immunol. 2018;9:2306.

35. Cheng X, et al. Structure and interactions of the human programmed cell death 1 receptor. J Biol Chem. 2013;288(17):11771-11785.

36. Zak KM, et al. Structure of the complex of human programmed death 1, PD-1, and its ligand PD-L1. Structure. 2015;23(12):2341-2348.

37. Pentcheva-Hoang T, et al. Programmed death-1 concentration at the immunological synapse is determined by ligand affinity and availability. Proc Natl Acad Sci U S A. 2007;104(45):17765-17770.

38. Colli ML, et al. PDL1 is expressed in the islets of people with type 1 diabetes and is up-regulated by interferons- $\alpha$ and- $\gamma$ via IRF1 induction. EBioMedicine. 2018;36:367-375.

39. Wan B, et al. Aberrant regulation of synovial T cell activation by soluble costimulatory molecules in rheumatoid arthritis. $J$ Immunol. 2006;177(12):8844-8850.

40. Ng KW, et al. Soluble PD-L1 generated by endogenous retroelement exaptation is a receptor antagonist. Elife. $2019 ; 8: \mathrm{e} 50256$.

41. Haile ST, et al. Tumor cell programmed death ligand 1-mediated T cell suppression is overcome by coexpression of CD80. $J$ Immunol. 2011;186(12):6822-6829.

42. Chaudhri A, et al. PD-L1 binds to B7-1 only in cis on the same cell surface. Cancer Immunol Res. 2018;6(8):921-929.

43. Sugiura D, et al. Restriction of PD-1 function by cis-PD-L1/CD80 interactions is required for optimal T cell responses. Science. 2019;364(6440):558-566.

44. Zhao Y, et al. Antigen-presenting cell-intrinsic PD-1 neutralizes PD-L1 in cis to attenuate PD-1 signaling in T cells. Cell Rep. 2018;24(2):379-390.

45. Diskin B, et al. PD-L1 engagement on T cells promotes self-tolerance and suppression of neighboring macrophages and effector T cells in cancer. Nat Immunol. 2020;21(4):442-454

46. Bennett F, et al. Program death-1 engagement upon TCR activation has distinct effects on costimulation and cytokine-driven proliferation: attenuation of ICOS, IL-4, and IL-21, but not CD28, IL-7, and IL-15 responses. J Immunol. 2003;170(2):711-718. 
47. Kamphorst AO, et al. Rescue of exhausted CD8 T cells by PD-1-targeted therapies is CD28-dependent. Science. 2017;355(6332):1423-1427.

48. Mizuno R, et al. PD-1 primarily targets TCR signal in the inhibition of functional T cell activation. Front Immunol. 2019;10:630

49. Nishimura $\mathrm{H}$, et al. Immunological studies on PD-1 deficient mice: implication of PD-1 as a negative regulator for B cell responses. Int Immunol. 1998;10(10):1563-1572.

50. McKay JT, et al. PD-1 suppresses protective immunity to Streptococcus pneumoniae through a B cell-intrinsic mechanism J Immunol. 2015;194(5):2289-2299.

51. Francisco LM, et al. PD-L1 regulates the development, maintenance, and function of induced regulatory T cells. $J$ Exp Med. 2009;206(13):3015-3029.

52. Stathopoulou C, et al. PD-1 inhibitory receptor downregulates asparaginyl endopeptidase and maintains Foxp3 transcription factor stability in induced regulatory T cells. Immunity. 2018;49(2):247-263

53. Asano T, et al. PD-1 modulates regulatory T-cell homeostasis during low-dose interleukin-2 therapy. Blood. 2017;129(15):2186-2197.

54. Sage PT, et al. The receptor PD-1 controls follicular regulatory T cells in the lymph nodes and blood. Nat Immunol. 2013;14(2):152-161

55. Huang X, et al. PD-1 expression by macrophages plays a pathologic role in altering microbial clearance and the innate inflammatory response to sepsis. Proc Natl Acad Sci U S A. 2009;106(15):6303-6308.

56. Pesce $S$, et al. Identification of a subset of human natural killer cells expressing high levels of programmed death 1: A phenotyp ic and functional characterization. J Allergy Clin Immunol. 2017;139(1):335-346.

57. McCafferty J. Phage Display of Peptides and Proteins - A Laboratory Manual. Academic Press; 1996.

58. Li Y, et al. Directed evolution of human T-cell receptors with picomolar affinities by phage display. Nat Biotechnol. 2005;23(3):349-354

59. Pardon E, et al. A general protocol for the generation of Nanobodies for structural biology. Nat Protoc. 2014;9(3):674-693

60. Boulter JM, et al. Stable, soluble T-cell receptor molecules for crystallization and therapeutics. Protein Eng. 2003;16(9):707-711.

61. Dolton G, et al. Optimized peptide-MHC multimer protocols for detection and isolation of autoimmune T-cells. Front Immunol. 2018;9:1378. 